\title{
Relativistic Band Calculation and the Optical Properties of Gold
}

\author{
Christensen, N Egede; Seraphin, B. 0.
}

Published in:

Physical Review B Condensed Matter

Link to article, DOI:

10.1103/PhysRevB.4.3321

Publication date:

1971

Document Version

Publisher's PDF, also known as Version of record

Link back to DTU Orbit

Citation (APA):

Christensen, N. E., \& Seraphin, B. O. (1971). Relativistic Band Calculation and the Optical Properties of Gold. Physical Review B Condensed Matter, 4(10), 3321-3344. https://doi.org/10.1103/PhysRevB.4.3321

\section{General rights}

Copyright and moral rights for the publications made accessible in the public portal are retained by the authors and/or other copyright owners and it is a condition of accessing publications that users recognise and abide by the legal requirements associated with these rights.

- Users may download and print one copy of any publication from the public portal for the purpose of private study or research.

- You may not further distribute the material or use it for any profit-making activity or commercial gain

- You may freely distribute the URL identifying the publication in the public portal

If you believe that this document breaches copyright please contact us providing details, and we will remove access to the work immediately and investigate your claim 


\title{
Relativistic Band Calculation and the Optical Properties of Gold ${ }^{\dagger}$
}

\author{
N. Egede Christensen \\ Physics Laboratory I, The Technical University of Denmark, 2800 Lyngby, Denmark \\ and \\ B. O. Seraphin \\ Optical Sciences Center, University of Arizona, Tucson, Arizona 85721
}

(Received 8 April 1971)

\begin{abstract}
The energy band structure of gold is calculated by the relativistic augmented-plane-wave (RA PW) method. A nonrelativistic calculation is also presented, and a comparison between this and the RAPW results demonstrates that the shifts and splittings due to relativistic effects are of the same order of magnitude as the gaps (approximately $1 \mathrm{eV}$ ). Various integrated functions, density of states, joint density of states, and energy distributions of joint density of states are derived from the RAPW calculation. These functions are used in an interpretation of photoemission and static reflectance measurements. It is shown that the photoemission results are extremely well described in terms of a model assuming all transitions to be direct whereas a nondirect model fails. The $\epsilon_{2}$ profile calculated in a crude model assuming constant matrix elements matches well the corresponding experimental results. The calculated interband edge $\left(\hbar \omega_{i}=2.38 \mathrm{eV}\right)$ agrees with experimental values, and the absorption tail below the interband edge which is found in experimental traces is also contained in the theoretical curve. By means of a calculation of the Fermi surface and the constant-energy-difference surfaces it has been possible to trace out the regions in $\vec{k}$ space where the edge and tail transitions occur. It is demonstrated that structure in the static reflection curves are not related to critical points in the band structure. The arguments are supported by calculations of temperature shifts of the critical-point energies and comparison to the observed temperature shifts of the elements of structure in the experimental $\epsilon_{2}$ function. Such structure may originate in extended rather than localized regions of $\vec{k}$ space. In contrast, critical-point transitions show up clearly in modulated reflectance spectra, and all elements of structure are fully accounted for by our band model. The temperature and strain responses in the band structure are determined by performing the RAPW calculation with two lattice constants and estimating the effects of the lattice vibrations by means of an OPW-LCAO (linear combination of atomic orbitals) scheme with pseudopotential Fourier constants reduced by the appropriate Debye-Waller factors. The phonon spectrum has been calculated for the latter purpose.
\end{abstract}

\section{INTRODUCTION}

The physical properties of the transition and noble metals are determined largely by the outermost $d$ electrons in the atoms. As this $d$ shell is progressively filled through a group of transition metals, the physical properties vary drastically. The noble metals follow right after the transition metals and have the $d$ shell filled. Although covering only energies below the Fermi level, the $d$ bands in the noble metals are still located in the region of band energies, and they strongly influence the band structure and the related physical properties. This implies that the band structure of the noble metals cannot be described in terms of a weak-pseudopotential model, as for many simple metals such as sodium, potassium, aluminum, lead, etc. The $d$ states are so localized that they sense the strong core potential, and their hybridization with the free-electron-like $s p$ bands must be properly taken into account.

In a band calculation the two key parameters, width and position of the $d$ bands, respond sensi- tively to the particular choice of the crystal potential. The $d$ states can be considered as quasibound states in an effective potential consisting of the crystal potential plus the centrifugal term $l(l+1) / r^{2}$ appearing in the radial Schrödinger equation. This effective potential exhibits a barrier, the size of which determines the lifetime of the virtually bound states, and through this barrier the width of the $d$ bands. The size of the barrier and therefore the width of the $d$ bands depends strongly on the particular choice of the potential. ${ }^{1}$ Similar considerations apply to the position of the $d$ bands as described by their center of gravity, the location of which can differ appreciably from the $d$ level of the isolated atom and is closer to the $d$ level corresponding to an atomic potential renormalized according to crystal-potential parameters. ${ }^{2}$

The strong sensitivity of width and position of the $d$ bands to small variations of the crystal potential has repercussions for the resulting band structure in general. The hybridization between the $d$ states and the $s p$ states manifests itself not only at the 
intersection of the $d$ bands and the $s p$ bands but also above the Fermi level, where it shifts the energy gaps at the zone faces with respect to the values obtained from a pseudopotential model for the conduction bands alone. In the case of the noble metals, the energy bands directly at the Fermi level are not very sensitive to the choice of potential. A potential yielding incorrect $d$ bands may still lead to a Fermi surface that explains most of the experimental data satisfactorily. True, the derivatives of the bands at the Fermi level as expressed in effective masses and group velocities depend on the width of the $d$ bands and their distance. from the Fermi level. However, because insufficient information on the actual electron-electron and electron-phonon enhancement introduces a rather wide margin in the expected degree of agreement between theory and experiment, these quantities will not be very discriminative for the potential. As a consequence, a sensitive check on the theoretical model results only from a comparison with experiments that monitor large energy ranges of the band structure, such as optical experiments.

It follows that, for the noble metals, more than usual attention must be paid to the proper choice of the potential. An empirical aspect enters here, and this reduces the $a b$ initio character of a firstprinciples calculation such as the augmented-planewave (APW) method, for instance. After all, the best ad hoc potential is the one that generates the closest agreement with properly chosen and interpreted experimental data. Following this guideline, a previous study of silver had examined a wide spectrum of various potentials. Optimal agreement with respect to width and position of the $d$ bands as derived from experiment was obtained from a model based on Dirac-Slater atomic wave functions and with full Slater exchange included in the atomic as well as in the crystal potential. The potential in this work was constructed in the same way as the best silver potential.

Since we place much emphasis on the choice of a potential that brings the resulting band structure in line with properly interpreted experimental data, we must define our terms in this respect. In contrast to Fermi-surface experiments, optical experiments involving excitations from states far below the Fermi level are easily misinterpreted. In correlating the calculated band separations and the calculated spectral functions to the experimental results, we must consider the following aspects:

(i) Because the $d$ bands are narrow, we must investigate possible effects of a localization of the $d$ states. It must be discussed whether an excitation from these bands can be treated as being extended and therefore on the same level as excitations from the $s p$ bands. Aspects enter here that are related to the question of validity of Koopmans's theorem. (ii) In the same context, the problem of direct-

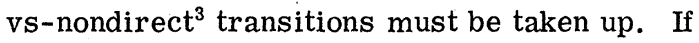
there is experimental evidence for nondirect transitions, caution must be exercised in comparing the calculated bands to such data. Occurrence of nondirect transitions indicates the presence of localization effects that restrict the interpretation on the basis of extended excitations. ${ }^{4,5}$

(iii) Special attention must be paid to an interpretation of static and modulated reflectance spectra in terms of the energy band structure. The dichotomy of localized-vs-extended excitations applies here with respect to critical points and the Brillouin zone. It must be recalled that the $\epsilon_{2}$ spectrum derived from static reflectance samples the sum of all interband transitions possible at a given photon energy ${ }^{6,7}$ Structure in this spectrum relates to the spectral profile of excitations summed over extended regions of $\overrightarrow{\mathrm{k}}$ space. ${ }^{8}$ In a rough picture assuming direct transitions with constant matrix elements we therefore must compare the experimental result to a theoretical quantity containing this sum of all contributions-the joint density-ofstates function. Only the $\Delta \epsilon_{2}$ spectrum derived from modulated reflectance experiments can be expected to clearly display contributions from localized regions in the Brillouin zone, such as critical points in the band structure.

Thus, the purpose of the present paper is not only to present a calculation of the energy bands of gold and to justify our belief in its accuracy by comparison to some measured excitation energies. We also examine the rules according to which the optical experiments are to be interpreted. For instance, a comparison to photoemission experiments involves two questions: (i) Does our calculated band structure predict the right values of $d$ band width and position? (ii) Is it possible to decide whether nondirect transitions give essential contributions or not? The actual answers to these questions are essential not only in a discussion of the reliability of the calculation treating the $d$ bands and the conduction band similarly, but they will also have consequences for the interpretation of the reflection experiments. The article then naturally contains three main sections, numerical band calculation, interpretation rules for optical experiments, and application of these rules to the case of gold and conclusions about the nature of the optical transitions in gold.

The crystal potential and justification of the actual choice are discussed in Sec. II. At the time a preliminary account ${ }^{9}$ of the present work was submitted, no other relativistic results were available for gold. To compare our potential model to those by other authors, and also to demonstrate the large relativistic effects in the gold bands, a preliminary nonrelativistic band structure is presented (Sec. 
IIIA). Section III is divided into five subsections and contains most of the results of the calculation of eigenvalues and derived spectral functions (density of states, joint density of states, and Fermi level). In order to make a full comparison to experiments, it was necessary to calculate the responses in the band structure due to changes in strain and temperature. The strain responses were determined by performing the RAPW calculation for two lattice constants, one corresponding to normal volume and another corresponding to an expanded lattice. The lattice expansion alone is not sufficient to simulate temperature shifts. Also the thermal lattice vibrations may introduce shifts, at least through a smearout of the potential. The effects of this smearout were estimated for the $s p$ bands by multiplying the pseudopotential Fourier constants in a combined interpolation scheme by the proper Debye-Waller factors. To evaluate the Debye-Waller factors it was necessary to calculate the phonon frequencies and phonon spectrum.

In Sec. IV, we discuss the rules for interpreting the results of photoemission, static reflectance, and modulated reflectance experiments. The interpretation of the three types of experiments is dealt with in Sec. V. We consider two simple models for photoemission. One is based exclusively on nondirect transitions, and the other assumes all transitions to be direct. Recently published ${ }^{10}$ polarimetric static reflectance work covers a large temperature range and is therefore well suited for comparison with our calculated temperature responses. Further, the calculation of the strain responses provides a strong support of our assignment of some structure elements in the thermoreflectance spectrum, for it shows that this assignment is consistent with piezoreflectance spectra.

\section{CRYSTAL POTENTIAL}

The crystal potential was approximated by the usual muffin-tin model. The Coulomb and exchange parts were treated separately, the former being a superposition of atomic Coulomb potentials and the latter chosen to be proportional to the cubic root of the density. The atomic Coulomb potentials were obtained by solving Poisson's equation with the atomic charge densities calculated by Liberman et al. ${ }^{11}$ Contributions from neighboring sites were, by application of Löwdin's $\alpha$-expansion technique, summed to give the spherically symmetric part of the Coulomb potential within the muffin-tin spheres. The constant Coulomb potential between the muffintin spheres was calculated by taking the Coulomb discontinuity at $R_{s}$ to be half the value given by Ewald's method. This leads to a somewhat larger discontinuity at the sphere than does the simple Wigner-Seitz sphere averaging. The exchange term in the same region was calculated by the usual $\rho^{1 / 3}$ approximation, inserting $\rho_{c}=\left(Z-N_{s}\right) /\left(\Omega_{c}-\Omega_{s}\right)$ for the average density in the interspherical region, where $Z$ is the atomic number, $N_{s}$ the number of electrons contained in a muffin-tin sphere, $\Omega_{c}$ the cell volume, and $\Omega_{s}$ the volume of the muffin-tin sphere.

As mentioned in the Introduction, a variety of potential functions can be generated by varying the exchange weight factor, i.e., by choosing different values of $c$ in $V_{\mathrm{ex}}=-6 c[(3 / 8 \pi) \rho(\overrightarrow{\mathrm{r}})]^{1 / 3}(\mathrm{Ry})$.

The procedure of varying the amount of Slater exchange in an ad hoc muffin-tin potential does not represent numerical investigation of the theoretical problem whether to use full Slater exchange or the reduced Gaspar-Kohn-Sham exchange, which is $\frac{2}{3}$ of the Slater term. The density functional formalism leading to the Kohn-Sham local approximations for exchange and correlation potentials refers to the ground state of the electron system and cannot be expected to hold in cases where in fact excitation spectra are considered. We consider the variation of the exchange weight factor merely as a simple way of generating different potentials.

In the case of silver, the responses in the band structure due to such variations were examined. ${ }^{12}$ An increase in $c$ will produce more tightly bound states, i. e., a narrowing of the $d$ bands, and move the $d$ bands toward lower energies. Figure 1 shows how the position and width of the $d$ bands vary $c$. The full lines correspond to potentials derived from relativistic Dirac-Slater atomic-charge densities calculated with $c=1$ in the atomic potential. The broken lines give the results of variation of the ex-

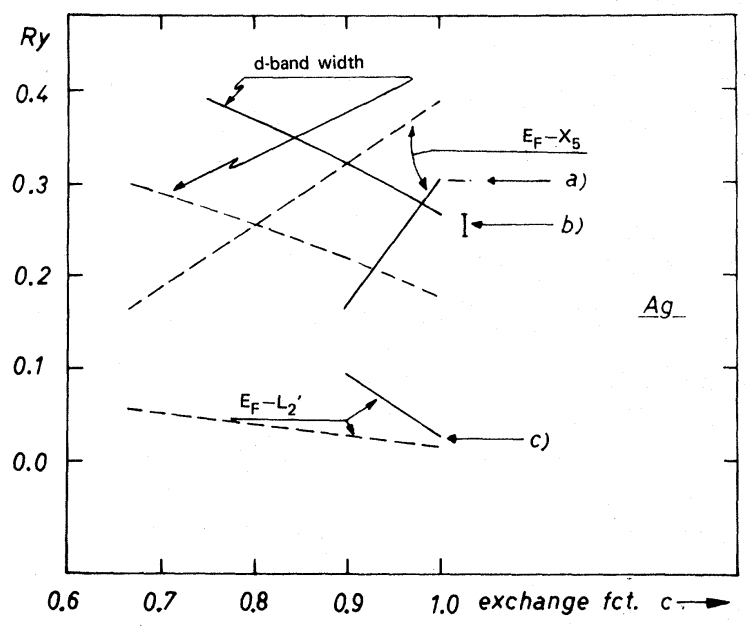

FIG. 1. Ag. Width of the $d$ band and the band separations $E_{F}-X_{5}, E_{F}-L_{2}^{\prime}$ calculated with different potentials generated by varying the amount $c$ of Slater exchange. Full curves: Dirac-Slater atomic potential $(c=1)$. Broken lines: Hartree-Fock-Slater with $c$ varying according to $c$ in the crystal potential. Arrows (a) - (c) indicate experimental results. 
change factor in the crystal potential as well as in the atomic potential. (Further, the atomic calculation is here nonrelativistic.) The changes in the band structure are more pronounced when $c$ is varied in the crystal potential alone than in the case where the atomic and crystal exchange terms are altered simultaneously.

In the case of silver, the potential based on relativistic Dirac-Slater densities and having $c=1$ led to a band structure in agreement with experiments in the following sense. The position and width of the $d$ bands agree with the photoemission results obtained by Berglund and Spicer. ${ }^{3}$ Agreement in position and width of the $d$ band is obtained if the hump in the density of states derived from the photoemission spectrum ${ }^{3}$ is assumed to give the threshold energy of emission from the $d$ bands. We do not have to make any assumptions about the particular type of transition, i.e., whether the transitions are "direct" or "nondirect." Using the silver potential selected in this way, the Fermi surface and related physical properties were calculated and found to agree well with a large variety of experiments. ${ }^{12,13}$ Thus, it was possible, even by using the same potential for the wide $s p$ bands and the narrow $d$ bands, to obtain a general agreement between theory and experiment. This could not be expected a priori because there might be considerable effects from the localization of the $d$ states.

If it is true that the $d$ states of silver are so nonlocalized that they can be treated in the same way as the conduction bands, then it is not less true in the case of gold, because the $\mathrm{Au} d$ bands are considerably broader than those of silver. Therefore, it seems reasonable to try to apply to gold the method of potential construction that led to the best $\mathrm{Ag}$ band structure.

The RAPW calculation for gold was performed with two lattice constants, 7.6813 and 7.7820 a. u. , the former corresponding to $T$ equal to absolute zero and the latter to a volume increased by $\Delta \mathrm{V} / \mathrm{V}$ $=3.9 \%$. The muffin-tin radius was 2.5857 a. u. ; i. e., the spheres do not touch each other. In the normal-volume case the constant potential between the spheres was $V_{0}=-1.1513$ Ry relative to vacuum. The major contribution to this comes from the exchange term $V_{\text {ex, out }}=-0.8255 \mathrm{Ry}$. The discontinuity $\Delta V_{0}$ of the potential at the muffin-tin radius is $0.1179 \mathrm{Ry}$. For the expanded lattice, $V_{0}=-1.0317$ Ry, $\Delta V_{0}=0.1179 \mathrm{Ry}$, and $V_{\text {ex, out }}=-0.8094 \mathrm{Ry}$. Because the generation of the potential functions is trivial, we have not included tables of the potentials. They can be obtained on request.

\section{RESULTS OF BAND CALCULATIONS}

\section{A. Nonrelativistic APW}

Eigenvalues at symmetry lines in the Brillouin zone (see Fig. 2) were obtained by application of

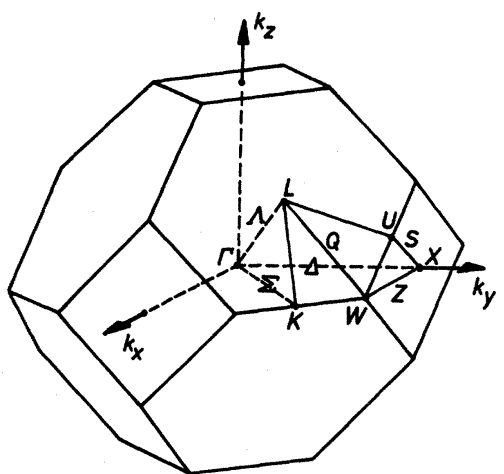

FIG. 2. Symmetry points in the fcc Brillouin zone and the irreducible $\frac{1}{48}$ zone.

symmetrized APW's. The advantages of symmetrization are obvious in this case. The computing time is reduced, and the irreducible representations can be identified.

Figure 3 shows the nonrelativistic bands. The qualitative features are as in the other noble metals, but gold has by far the widest $d$ bands. The $d$ band width is approximately $0.420 \mathrm{Ry}$ for gold, whereas for silver it is approximately $0.260 \mathrm{Ry}$. Table I gives some band separations and compares them to previous calculations. Energy differences involving the Fermi level may be in error at the second decimal place owing to the rough method of estimating $E_{F}$ as described in the footnote to Table I. Further, it should be noted that different lattice parameters were used in the five calculations compared in Table I. The present calculation seems to agree rather well with the Korringa-Kohn-Rostokew (KKR) results obtained by Ballinger and Marshall. ${ }^{14}$ They used a Gasper potential with reduced exchange. Kupratakuln and Fletcher ${ }^{15}$ based their APW calculation on Herman and Skillman's ${ }^{16}$ atomic wave functions. This was also done in the work by O'Sullivan, Switendick, and Schirber. ${ }^{17}$ Jacobs $^{18}$ constructed a nonlocal potential.

\section{B. Relativistic Au Band: Normal Volume}

The necessity of including relativistic effects when determining the electronic states of a solid becomes pronounced when the probability amplitudes for the electrons are large in regions of space where their velocity $v$ is a non-negligible fraction of the velocity of light $c$. This region where $v / c$ is not $\ll 1$ increases in extension with increasing nuclear charge $Z e$. From atomic calculations (e.g., Herman and Skillman ${ }^{16}$ ) it is well known that the nuclear charge of gold is so large $(Z=79)$ that the relativistic effects are quite important. Shifts of the same order of magnitude must therefore be expected also in the solid. It is, however, of great importance to the practical calculation to notice that the rel- 


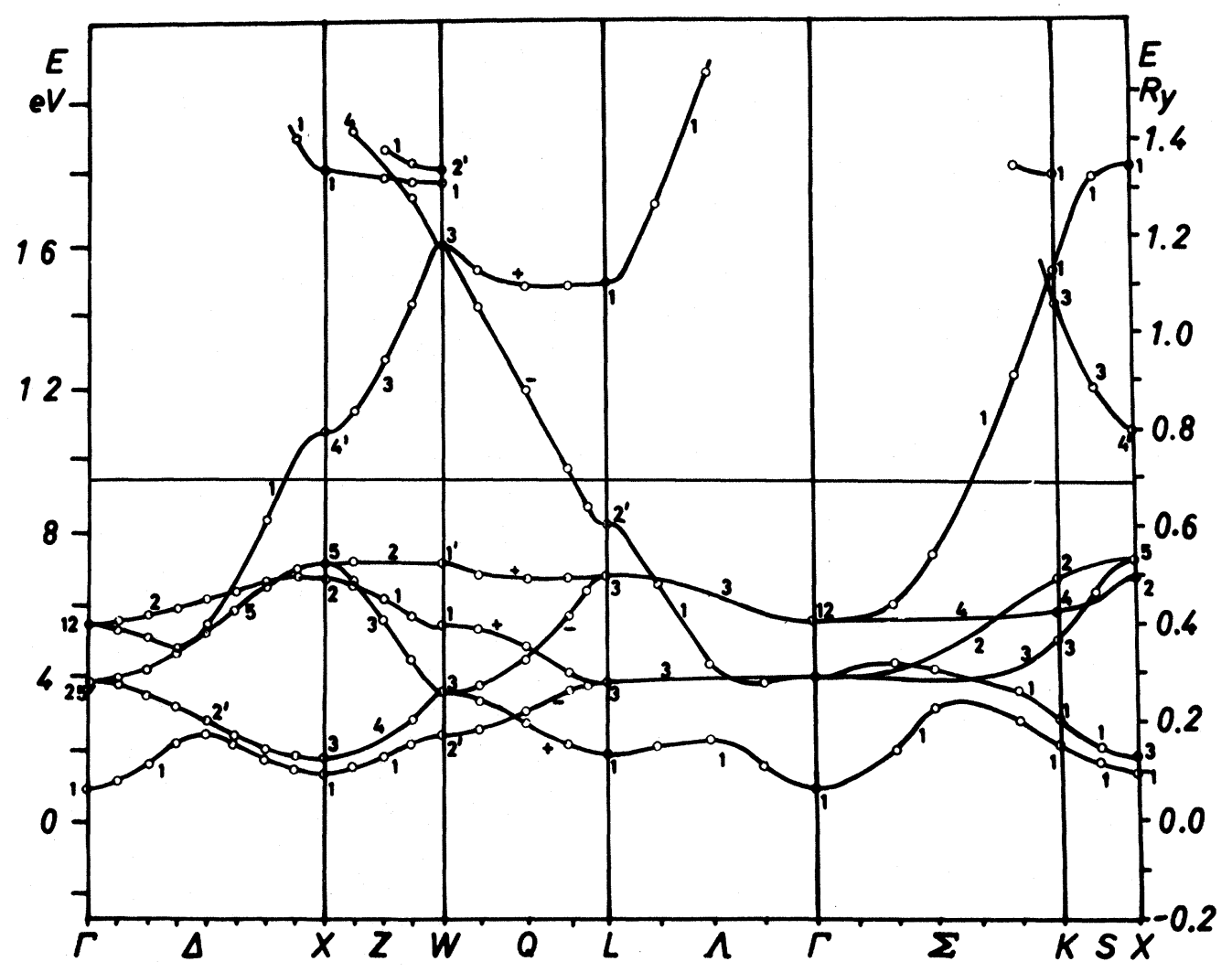

FIG. 3. APW energy bands (nonrelativistic) of gold (normal volume).

TABLE I. Band separations in gold. Nonrelativistic APW. Energies are in Ry, and column a is from Ballinger and Marshall (Ref. 14); b, Jacobs (Ref. 18); c, Kupratakuln and Fletcher (Ref. 15); and d, O'Sullivan, Switendick, and Schirber (Ref. 17). In the calculation b, $E_{F}$ was chosen to give $E_{F}-L_{2}^{\prime}$ in agreement with experiments. All numbers in column c, except $L_{2}^{\prime}-L_{3}$, were read from Fig. 1 in Ref. 15. In the present work (last column), the Fermi level was chosen to provide the Fermi-surface dimensions $k_{100}$ and $k_{110}$ approximately as determined from the RAPW Fermi-surface calculations (Ref. 29).

\begin{tabular}{lccccc}
\hline \hline & & & & & This \\
& $\mathrm{a}$ & $\mathrm{b}$ & $\mathrm{c}$ & $\mathrm{d}$ & work \\
\hline$\Gamma_{25}^{\prime}-\Gamma_{1}$ & 0.19 & 0.191 & 0.06 & 0.086 & 0.222 \\
$X_{4}^{\prime}-X_{5}$ & 0.31 & 0.348 & 0.46 & $\cdots$ & 0.273 \\
$X_{5}-X_{1}$ & 0.41 & 0.278 & 0.30 & 0.315 & 0.430 \\
$L_{1}-L_{2}^{\prime}$ & 0.44 & 0.368 & 0.37 & $\cdots$ & 0.501 \\
$X_{5}-X_{1}$ & 0.42 & 0.355 & 0.24 & 0.274 & 0.461 \\
$W_{3}-W_{1}^{\prime}$ & 0.69 & $\cdots$ & 0.82 & $\cdots$ & 0.657 \\
$E_{F}-L_{3}^{\prime}$ & 0.22 & 0.232 & 0.33 & 0.322 & 0.22 \\
$E_{F}-X_{5}$ & 0.20 & 0.210 & 0.31 & 0.301 & 0.19 \\
$E_{F}-L_{2}^{\prime}$ & 0.06 & 0.029 & 0.02 & 0.054 & 0.11 \\
$E_{F}-\Gamma_{1}$ & 0.61 & $\cdots$ & 0.57 & 0.575 & 0.63 \\
$L_{2}^{\prime}-L_{3}$ & $\cdots$ & $\cdots$ & 0.306 & $\cdots$ & 0.226 \\
Lattice & & & & & \\
parame- & 7.707 & 7.6799 & 7.706 & 7.72056 & 7.6813 \\
ter (a.u.) & & & & & \\
\hline \hline
\end{tabular}

ativistic corrections are not equally important in all regions of the unit cell. Even when $Z$ is as large as in gold, $v / c$ is much less than one in the region between the muffin-tin spheres $\left(r>R_{s}\right){ }^{19}$ This means that we can neglect the small components of the Dirac wave function for $r>R_{s}$ and retain in this region simple plane-wave representation of the expansion functions (RAPW's), one for each spin.

The fact that relativistic effects need be included only for $r<R_{s}$ has important consequences: (i) It becomes possible to make the $\left(r<R_{s}\right)$ and the $\left(r>R_{s}\right)$ parts of each RAPW join up at the muffintin sphere. (ii) The APW matrix has the same form whether the Schrödinger, Pauli, or Dirac Hamiltonian are employed ${ }^{20,21}$ :

$$
\begin{aligned}
M_{i j}= & \left(\overrightarrow{\mathrm{k}}_{i} \cdot \overrightarrow{\mathrm{k}}_{j}-E\right) U\left(\left|\overrightarrow{\mathrm{k}}_{i}-\overrightarrow{\mathrm{k}}_{j}\right|\right)\left\langle m_{i} \mid m_{j}\right\rangle+\frac{4 \pi R_{s}^{2}}{\Omega_{c}} \\
& \times \sum_{i=0}^{\infty} P_{l}\left(\hat{k}_{i} \cdot \hat{k}_{j}\right) j_{l}\left(k_{i} R_{s}\right) j_{l}\left(k_{j} R_{s}\right) \alpha_{l}(E)\left\langle m_{i} \mid m_{j}\right\rangle \\
+ & \frac{4 \pi R_{s}^{2}}{\Omega_{c}} \sum_{l=1}^{\infty} P_{l}^{\prime}\left(\hat{k}_{i} \cdot \hat{k}_{j}\right) j_{l}\left(k_{i} R_{s}\right) j_{l}\left(k_{j} R_{s}\right) \beta_{l}(E) \\
& \times\left\langle m_{i}\left|i \vec{\sigma} \cdot\left(\hat{k}_{j} \times \hat{k}_{i}\right)\right| m_{j}\right\rangle
\end{aligned}
$$


The vectors $\overrightarrow{\mathrm{k}}_{i}$ are sums of the considered $\overrightarrow{\mathrm{k}}$ and reciprocal lattice vectors $\overrightarrow{\mathrm{K}}_{i}, \overrightarrow{\mathrm{k}}_{i}=\overrightarrow{\mathrm{k}}+\overrightarrow{\mathrm{K}}_{i}$, and $m_{i}$ is the spin associated with the plane-wave part of the RAPW. $U(\vec{q})$ is the Fourier transform of a step function that has the value 1 for $r>R_{s}$ and 0 for $r<R_{s} . \quad P_{l}$ and $j_{l}$ are the Legendre polynomials and spherical Bessel functions, respectively. The Pauli spin matrix is denoted by $\vec{\sigma}$. Only the quantities $\alpha_{l}(E)$ and $\beta_{l}(E)$ depend on which Hamiltonian is used:

Schrödinger equation:

$$
\alpha_{l}(E)=(2 l+1) \frac{R_{l}^{\prime}}{R_{l}}, \quad \beta_{l}(E)=0
$$

Pauli:

$$
\begin{aligned}
& \alpha_{l}(E)=\left.(l+1) \frac{R_{k}^{\prime}}{R_{k}}\right|_{k=-l-1}+\left.l \frac{R_{k}^{\prime}}{R_{k}}\right|_{k=l}, \\
& \beta_{l}(E)=\left.\frac{R_{k}^{\prime}}{R_{k}}\right|_{k=-l-1}-\left.\frac{R_{k}^{\prime}}{R_{k}}\right|_{k=l} ;
\end{aligned}
$$

Dirac:

$$
\begin{aligned}
& \alpha_{l}(E)=\left.(l+1) \frac{c f_{k}}{g_{k}}\right|_{k=-l-1}+\left.l \frac{c f_{k}}{g_{k}}\right|_{k=\imath}, \\
& \beta_{l}(E)=\left.\frac{c f_{k}}{g_{k}}\right|_{k=-l-1}-\left.\frac{c f_{k}}{g_{k}}\right|_{k=l}+\frac{2 l+1}{R_{s}} .
\end{aligned}
$$

The functions $R_{l}(E, r), R_{k}(E, r), f_{k}(E, r)$, and $g_{k}(E, r)$ are the solutions of the radial Schrödinger, Pauli, and Dirac equations. ${ }^{22}$ The quantum number $\kappa$ is the eigenvalue ${ }^{22}$ of $-(\vec{\sigma} \cdot \vec{i}+1)$. As mentioned by Andersen, ${ }^{20}$ the Dirac equations for $\alpha_{\imath}$ and $\beta_{t}$ for large $r$ (the functions $\alpha_{l}$ and $\beta_{l}$ are evaluated for $r=R_{s}$ ) become equivalent to the Pauli expressions. It is then reasonable to discuss the relativistic effects in the language of the Pauli theory, i.e. , in terms of mass-velocity, Darwin, and spin-orbit corrections. These three correction terms can be identified after a Foldy-Wouthuysen transformation of the Dirac equation neglecting terms smaller than those of second order in $1 / c{ }^{20,22}$ The massvelocity term describes the change in energy related to the difference between the rest mass and the mass moving electron. In the atom, ${ }^{16}$ this term lowers the $5 d$ level by approximately $0.15 \mathrm{Ry}$ and the $6 s$ level by approximately $0.34 \mathrm{Ry}$. The Darwin term originates in the oscillatory component of the electron motion. In a Coulomb potential it is only nonzero for the states having nonvanishing wave functions at $r=0$, i. e., for the $s$ states. It is therefore not surprising that the Darwin shift of the atomic $d$ level is considerably smaller than that of the $s$ level: For $5 d$ it is approximately 0.002 Ry (lowering), whereas the $6 s$ level is raised by approximately $0.190 \mathrm{Ry}$. We would then expect the bottom of the band structure $\left(\Gamma_{6}^{+}\right)$in the RAPW calculation to lie approximately at $\Delta E=(0.34-0.19)=0.15$ Ry below the $\Gamma_{1}$ level found in the APW scheme.

The spin-orbit term is particularly interesting in connection with the band structure because this is the only term among the three corrections that splits the levels. For a central field these splittings are related to the spin-orbit parameter $\frac{1}{2} \xi_{n, l}$. Using the values for this parameter obtained in the atomic calculation, we can estimate the expected order of magnitude of the spin-orbit splittings $\Delta E_{\text {so }}$ at $\Gamma$ and $X:$ From the calculation by Herman and Skillman ${ }^{16}$ the spin-orbit parameter for the $5 d$ level is

$$
\frac{1}{2} \xi_{5 d} \approx 0.026 \mathrm{Ry} \text {. }
$$

A rough estimate of $\Delta E_{\mathrm{so}}$ then yields

$$
\begin{array}{ll}
\Gamma_{25}^{\prime} \rightarrow \Gamma_{8}^{+}+\Gamma_{7}^{+}, & \Delta E_{\mathrm{so}} \approx \frac{3}{2} \xi_{5 d} \approx 0.08 \mathrm{Ry}, \\
X_{5} \rightarrow X_{6}^{+}+X_{7}^{+}, & \Delta E_{\mathrm{so}} \approx \xi_{5 d} \approx 0.05 \mathrm{Ry} .
\end{array}
$$

This calculation is of course very rough since the spin-orbit parameter in the solid may differ appreciably from that of the atom. It exhibits appreciable variations with energy within the $d$ band. ${ }^{20}$ However, the discussion above serves to characterize the relativistic effects, and the estimates show that these shifts are of the same order of magnitude as the band gaps, and they are therefore very important in a band calculation that is compared to optical experiments.

The energy band structure for gold was calculated by determining nine RAPW energy values at each of 89 points $\vec{k}$ uniformly distributed but confined to

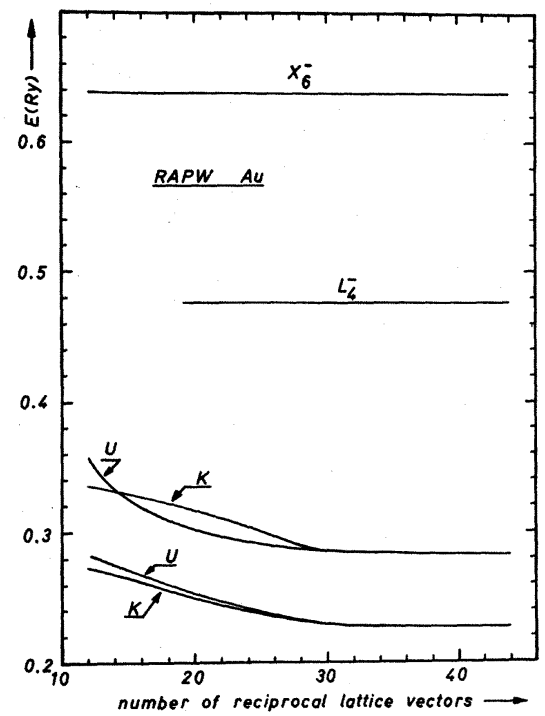

FIG. 4. Examples from the convergence test of the RAPW expansion. Energies at $U$ and $K$ must be identical since these points coincide when the zones are stacked. 
TABLE II. Relativistic APW eigenvalues. Normal volume. The energies are measured in Ry from the constant potential energy $V_{0}$ between the muffin-tin spheres. $V_{0}=-1.1513 \mathrm{Ry}, E_{F}=0.530 \mathrm{Ry}$.

\begin{tabular}{|c|c|c|c|c|c|c|c|c|c|c|}
\hline & $\overrightarrow{\mathrm{k}} / \frac{\pi}{4 a}$ & $\begin{array}{c}\text { Band } \\
1\end{array}$ & $\begin{array}{c}\text { Band } \\
2\end{array}$ & $\begin{array}{c}\text { Band } \\
3\end{array}$ & $\begin{array}{c}\text { Band } \\
4\end{array}$ & $\begin{array}{c}\text { Band } \\
5\end{array}$ & $\begin{array}{c}\text { Band } \\
6\end{array}$ & $\begin{array}{c}\text { Band } \\
7\end{array}$ & $\begin{array}{c}\text { Band } \\
8\end{array}$ & $\begin{array}{c}\text { Band } \\
9\end{array}$ \\
\hline$\Gamma$ & $\begin{array}{lll}0 & 0 & 0\end{array}$ & -0.1641 & 0.1150 & 0.1150 & 0.2110 & 0.2880 & 0.2880 & 1.6755 & 1.8555 & 1.8555 \\
\hline$\Delta$ & 010 & -0.1507 & 0.1111 & 0.1158 & 0.2125 & 0.2810 & 0.2895 & 1.6900 & 1.8286 & 1.8500 \\
\hline$\Delta$ & 020 & -0.1122 & 0.1042 & 0.1248 & 0.2202 & 0.2693 & 0.2961 & 1.7304 & 1.7654 & 1.8234 \\
\hline$\Delta$ & 030 & -0.0550 & 0.0898 & 0.1375 & 0.2371 & 0.2628 & 0.3087 & 1.6828 & 1.7381 & 1.7880 \\
\hline$\Delta$ & 040 & -0.0010 & 0.0697 & 0.1633 & 0.2618 & 0.2763 & 0.3279 & 1.5488 & 1.6972 & 1.7038 \\
\hline$\Delta$ & 050 & 0.0149 & 0.0487 & 0.2268 & 0.2878 & 0.3206 & 0.3532 & 1.4180 & 1.5550 & 1.5880 \\
\hline$\Delta$ & 060 & 0.0043 & 0.0309 & 0.2904 & 0.3090 & 0.3826 & 0.4163 & 1.2300 & 1.3970 & 1.4700 \\
\hline$\Delta$ & 070 & -0.0085 & 0.0191 & 0.3221 & 0.3267 & 0.4074 & 0.5523 & 1.0596 & 1.2889 & 1.3875 \\
\hline$X$ & 080 & -0.0134 & 0.0151 & 0.3266 & 0.3392 & 0.4175 & 0.6384 & 0.9562 & 1.2571 & 1.3612 \\
\hline \multirow[t]{7}{*}{$\Sigma$} & 110 & -0.1378 & 0.1110 & 0.1168 & 0.2147 & 0.2777 & 0.2898 & 1.6993 & 1.7646 & 1.8916 \\
\hline & 120 & -0.1003 & 0.1052 & 0.1239 & 0.2231 & 0.2668 & 0.2977 & 1.6676 & 1.7231 & 1.8557 \\
\hline & 130 & -0.0447 & 0.0917 & 0.1341 & 0.2398 & 0.2606 & 0.3138 & 1.5790 & 1.6945 & 1.8400 \\
\hline & 140 & 0.0082 & 0.0731 & 0.1525 & 0.2635 & 0.2755 & 0.3391 & 1.4767 & 1.6051 & 1.7454 \\
\hline & 150 & 0.0238 & 0.0533 & 0.2013 & 0.2904 & 0.3153 & 0.3811 & 1.3544 & 1.4964 & 1.5749 \\
\hline & 160 & 0.0126 & 0.0363 & 0.2566 & 0.3127 & 0.3611 & 0.4662 & 1.2131 & 1.3681 & 1.4339 \\
\hline & 170 & -0.0005 & 0.0252 & 0.2942 & 0.3253 & 0.3915 & 0.5937 & 1.0495 & 1. 2711 & 1.3581 \\
\hline$Z$ & 180 & -0.0056 & 0.0213 & 0.3090 & 0.3282 & 0.4027 & 0.6815 & 0.9472 & 1.2395 & 1.3323 \\
\hline \multirow[t]{6}{*}{$\Sigma$} & 220 & -0.0663 & 0.1051 & 0.1248 & 0.2315 & 0.2574 & 0.3103 & 1.5547 & 1.6811 & 1.8834 \\
\hline & 230 & -0.0162 & 0.0971 & 0.1296 & 0.2453 & 0.2506 & 0.3364 & 1.4516 & 1.6069 & 1.8875 \\
\hline & 240 & 0.0324 & 0.0837 & 0.1352 & 0.2602 & 0.2698 & 0.3766 & 1.3526 & 1.5102 & 1.7482 \\
\hline & 250 & 0.0473 & 0.0680 & 0.1608 & 0.2882 & 0.3022 & 0.4402 & 1.2527 & 1.4061 & 1.5899 \\
\hline & 260 & 0.0350 & 0.0536 & 0.2019 & 0.3071 & 0.3425 & 0.5405 & 1.1490 & 1.3074 & 1.4139 \\
\hline & 270 & 0.0211 & 0.0442 & 0.2379 & 0.3120 & 0.3735 & 0.6726 & 1.0271 & 1.2278 & 1.3054 \\
\hline$Z$ & 280 & 0.0157 & 0.0410 & 0.2543 & 0.3101 & 0.3853 & 0.7749 & 0.9265 & 1.1969 & 1.2724 \\
\hline \multirow[t]{5}{*}{$\Sigma$} & 330 & 0.0229 & 0.0991 & 0.1322 & 0.2359 & 0.2568 & 0.3758 & 1.3393 & 1.5097 & 1.8510 \\
\hline & 340 & 0.0587 & 0.0948 & 0.1346 & 0.2403 & 0.2750 & 0.4303 & 1. 2423 & 1.4062 & 1.7589 \\
\hline & 350 & 0.0739 & 0.0862 & 0.1370 & 0.2682 & 0.3009 & 0.5074 & 1.1680 & 1.3100 & 1.5950 \\
\hline & 360 & 0.0619 & 0.0792 & 0.1567 & 0.2875 & 0.3346 & 0.6156 & 1.0876 & 1.2145 & 1.4311 \\
\hline & 370 & 0.0470 & 0.0743 & 0.1809 & 0.2889 & 0.3647 & 0.7506 & 1.0111 & 1.1513 & 1.2836 \\
\hline$Z$ & 380 & 0.0413 & 0.0726 & 0.1927 & 0.2858 & 0.3768 & 0.8793 & 0.9104 & 1.1276 & 1.2263 \\
\hline \multirow[t]{4}{*}{$\Sigma$} & 440 & 0.0717 & 0.0976 & 0.1557 & 0.2275 & 0.2880 & 0.4959 & 1.1558 & 1.2986 & 1.7716 \\
\hline & 450 & 0.0744 & 0.0964 & 0.1574 & 0.2464 & 0.3085 & 0.5793 & 1.0926 & 1.1957 & 1.6591 \\
\hline & 460 & 0.0689 & 0.0951 & 0.1527 & 0.2677 & 0.3357 & 0.6870 & 1.0463 & 1.1116 & 1.4733 \\
\hline & 470 & 0.0584 & 0.0980 & 0.1534 & 0.2733 & 0.3628 & 0.8110 & 1.0006 & 1.0685 & 1.3009 \\
\hline$W$ & 480 & 0.0538 & 0.0996 & 0.1549 & 0.2730 & 0.3747 & 0.8957 & 0.9629 & 1.0619 & 1.2130 \\
\hline \multirow[t]{3}{*}{$\Sigma$} & 550 & 0.0584 & 0.1017 & 0.1804 & 0.2465 & 0.3229 & 0.6613 & 1.0476 & 1.0968 & 1.6841 \\
\hline & 560 & 0.0478 & 0.0911 & 0.1886 & 0.2668 & 0.3433 & 0.7582 & 0.9815 & 1.0579 & 1.5185 \\
\hline & 570 & 0.0427 & 0.0778 & 0.1922 & 0.2815 & 0.3661 & 0.8532 & 0.9187 & 1.0757 & 1.3416 \\
\hline \multirow[t]{8}{*}{$K, U$} & 660 & 0.0287 & 0.0705 & 0.2270 & 0.2822 & 0.3576 & 0.8283 & 0.8912 & 1.0700 & 1.4957 \\
\hline & 111 & -0.1254 & 0.1127 & 0.1165 & 0.2173 & 0.2756 & 0.2903 & 1.6738 & 1.7331 & 1.9267 \\
\hline & 121 & -0.0895 & 0.1081 & 0.1227 & 0.2254 & 0.2664 & 0.2986 & 1.5833 & 1.7448 & 1.8877 \\
\hline & 131 & -0.0368 & 0.0965 & 0.1317 & 0.2377 & 0.2659 & 0.3162 & 1.4868 & 1.7550 & 1.9562 \\
\hline & 141 & 0.0128 & 0.0804 & 0.1460 & 0.2512 & 0.2929 & 0.3442 & 1.3854 & 1.6501 & 1.7384 \\
\hline & 151 & 0.0276 & 0.0617 & 0.1876 & 0.2736 & 0.3322 & 0.3966 & 1.2759 & 1.5136 & 1.5970 \\
\hline & 161 & 0.0177 & 0.0442 & 0.2401 & 0.2983 & 0.3625 & 0.4952 & 1.1605 & 1.3546 & 1.4683 \\
\hline & 171 & 0.0055 & 0.0327 & 0.2793 & 0.3141 & 0.3833 & 0.6263 & 1.0298 & 1.2286 & 1.3945 \\
\hline \multirow[t]{11}{*}{$S$} & 181 & 0.0006 & 0.0288 & 0.2954 & 0.3177 & 0.3914 & 0.7184 & 0.9350 & 1.1868 & 1.3600 \\
\hline & 221 & -0.0589 & 0.1114 & 0.1238 & 0.2307 & 0.2628 & 0.3091 & 1.4697 & 1.7288 & 1.8984 \\
\hline & 231 & -0.0156 & 0.1073 & 0.1309 & 0.2330 & 0.2696 & 0.3339 & 1.3614 & 1.6823 & 1.8374 \\
\hline & 241 & 0.0239 & 0.0990 & 0.1376 & 0.2403 & 0.2981 & 0.3750 & 1.2571 & 1.6022 & 1.7350 \\
\hline & 251 & 0.0383 & 0.0830 & 0.1611 & 0.2609 & 0.3313 & 0.4469 & 1.1564 & 1.4935 & 1.5933 \\
\hline & 261 & 0.0324 & 0.0648 & 0.1988 & 0.2850 & 0.3545 & 0.5584 & 1.0636 & 1.3488 & 1.4671 \\
\hline & 271 & 0.0224 & 0.0534 & 0.2329 & 0.2992 & 0.3692 & 0.6961 & 0.9788 & 1.1929 & 1.3998 \\
\hline & 281 & 0.0181 & 0.0498 & 0.2483 & 0.3014 & 0.3754 & 0.8033 & 0.9042 & 1.1221 & 1.3781 \\
\hline & 331 & 0.0115 & 0.1102 & 0.1456 & 0.2257 & 0.2745 & 0.3716 & 1.2456 & 1.5985 & 1.8093 \\
\hline & 341 & 0.0364 & 0.1062 & 0.1558 & 0.2291 & 0.2942 & 0.4300 & 1.1416 & 1.5099 & 1.7380 \\
\hline & 351 & 0.0524 & 0.0967 & 0.1549 & 0.2506 & 0.3215 & 0.5122 & 1.0497 & 1.4203 & 1.6072 \\
\hline
\end{tabular}


TABLE II (Continued).

\begin{tabular}{|c|c|c|c|c|c|c|c|c|c|c|}
\hline & $\overrightarrow{\mathrm{k}} / \frac{\pi}{4 a}$ & $\begin{array}{c}\text { Band } \\
1\end{array}$ & $\begin{array}{c}\text { Band } \\
2\end{array}$ & $\begin{array}{c}\text { Band } \\
3\end{array}$ & $\begin{array}{c}\text { Band } \\
4\end{array}$ & $\begin{array}{c}\text { Band } \\
5\end{array}$ & $\begin{array}{c}\text { Band } \\
6\end{array}$ & $\begin{array}{c}\text { Band } \\
7\end{array}$ & $\begin{array}{c}\text { Band } \\
8\end{array}$ & $\begin{array}{c}\text { Band } \\
9\end{array}$ \\
\hline & 361 & 0.0543 & 0.0850 & 0.1648 & 0.2722 & 0.3430 & 0.6310 & 0.9737 & 1.3238 & 1.4524 \\
\hline & 371 & 0.0466 & 0.0794 & 0.1823 & 0.2813 & 0.3588 & 0.7681 & 0.9232 & 1.1832 & 1.3641 \\
\hline & 381 & 0.0425 & 0.0781 & 0.1917 & 0.2816 & 0.3660 & 0.8535 & 0.9184 & 1.0758 & 1.3416 \\
\hline & 441 & 0.0478 & 0.1028 & 0.1820 & 0.2219 & 0.3014 & 0.4958 & 1.0435 & 1.4174 & 1.7445 \\
\hline & 451 & 0.0588 & 0.0955 & 0.1767 & 0.2399 & 0.3199 & 0.5956 & 0.9618 & 1.3500 & 1.6549 \\
\hline & 461 & 0.0669 & 0.0876 & 0.1650 & 0.2604 & 0.3392 & 0.7027 & 0.8983 & 1.2672 & 1.4827 \\
\hline \multirow[t]{9}{*}{$Q$} & 471 & 0.0622 & 0.0930 & 0.1567 & 0.2710 & 0.3555 & 0.8074 & 0.8917 & 1.1836 & 1.3423 \\
\hline & 551 & 0.0533 & 0.0975 & 0.1877 & 0.2426 & 0.3311 & 0.6742 & 0.8889 & 1.2652 & 1.6892 \\
\hline & 561 & 0.0483 & 0.0904 & 0.1858 & 0.2620 & 0.3460 & 0.7749 & 0.8358 & 1.2165 & 1.5294 \\
\hline & 222 & -0.0390 & 0.1168 & 0.1349 & 0.2285 & 0.2736 & 0.3134 & 1.3477 & 1.7247 & 1.9518 \\
\hline & 232 & -0.0121 & 0.1140 & 0.1564 & 0.2243 & 0.2914 & 0.3346 & 1.2318 & 1.6971 & 1.8563 \\
\hline & 242 & 0.0139 & 0.1101 & 0.1632 & 0.2274 & 0.3255 & 0.3813 & 1.1238 & 1.6625 & 1.7363 \\
\hline & 252 & 0.0307 & 0.0996 & 0.1684 & 0.2416 & 0.3492 & 0.4752 & 1.0220 & 1.5480 & 1.6546 \\
\hline & 262 & 0.0347 & 0.0843 & 0.1890 & 0.2607 & 0.3583 & 0.6051 & 0.9399 & 1.3790 & 1.5672 \\
\hline & 272 & 0.0310 & 0.0738 & 0.2142 & 0.2766 & 0.3587 & 0.7512 & 0.9000 & 1.1896 & 1.5133 \\
\hline \multirow[t]{8}{*}{$U, K$} & 282 & 0.0285 & 0.0706 & 0.2263 & 0.2823 & 0.3576 & 0.8283 & 0.8912 & 1.0700 & 1.4957 \\
\hline & 332 & -0.0032 & 0.1111 & 0.1950 & 0.2208 & 0.3034 & 0.3609 & 1.1114 & 1.6577 & 1.8177 \\
\hline & 342 & 0.0113 & 0.1076 & 0.1963 & 0.2319 & 0.3262 & 0.4299 & 1.0038 & 1.6211 & 1.7256 \\
\hline & 352 & 0.0289 & 0.1013 & 0.1862 & 0.2400 & 0.3453 & 0.5278 & 0.9096 & 1.5560 & 1.6300 \\
\hline & 362 & 0.0434 & 0.0932 & 0.1824 & 0.2507 & 0.3499 & 0.6673 & 0.8329 & 1.4056 & 1.5633 \\
\hline & 372 & 0.0481 & 0.0906 & 0.1854 & 0.2621 & 0.3461 & 0.7749 & 0.8357 & 1.2164 & 1.5293 \\
\hline & 442 & 0.0171 & 0.1043 & 0.1995 & 0.2507 & 0.3373 & 0.4941 & 0.9018 & 1.5634 & 1.7062 \\
\hline & 452 & 0.0308 & 0.0989 & 0.1953 & 0.2483 & 0.3394 & 0.6000 & 0.8280 & 1.5062 & 1.6482 \\
\hline \multirow[t]{2}{*}{$Q$} & 462 & 0.0503 & 0.0905 & 0.1842 & 0.2489 & 0.3436 & 0.7250 & 0.7612 & 1.4286 & 1.5288 \\
\hline & 552 & 0.0355 & 0.0977 & 0.1947 & 0.2455 & 0.3457 & 0.6796 & 0.7577 & 1.4505 & 1. 6962 \\
\hline \multirow[t]{5}{*}{$\Lambda$} & 333 & -0.0083 & 0.1078 & 0.2050 & 0.2686 & 0.3376 & 0.3544 & 0.9893 & 1.6323 & 1.8441 \\
\hline & 343 & -0.0023 & 0.1051 & 0.1996 & 0.2806 & 0.3515 & 0.4296 & 0.8780 & 1.6155 & 1.7720 \\
\hline & 353 & 0.0136 & 0.1022 & 0.1958 & 0.2626 & 0.3540 & 0.5536 & 0.8010 & 1.5870 & 1.6993 \\
\hline & 363 & 0.0355 & 0.0977 & 0.1947 & 0.2454 & 0.3458 & 0.6795 & 0.7577 & 1.4504 & 1.6962 \\
\hline & 443 & -0.0032 & 0.1032 & 0.1968 & 0.3031 & 0.3553 & 0.4865 & 0.7948 & 1.6008 & 1.7485 \\
\hline$Q$ & 453 & 0.0085 & 0.1019 & 0.1956 & 0.2813 & 0.3521 & 0.5709 & 0.7528 & 1.5946 & 1.6885 \\
\hline$L$ & 444 & -0.0100 & 0.1025 & 0.1963 & 0.3235 & 0.3760 & 0.4773 & 0.7512 & 1.5923 & 1.7775 \\
\hline
\end{tabular}

the irreducible $\frac{1}{48}$ Brillouin zone (Table II). The maximum value of $\kappa$ included [Eq. (1)] was chosen to be 9 in the $\alpha$ terms and 5 in the more rapidly converging $\beta$ series. After examining the convergence with respect to the number of RAPW-expansion functions (Fig. 4), we decided to include 88 RAPW's, i.e., 44 reciprocal lattice vectors. This ensured convergence to less than $0.001 \mathrm{Ry}$ even for the excited states. The basic vectors were pointing into the solid angle defined by inverting the $\frac{1}{48}$ zone to which $\overrightarrow{\mathrm{k}}$ was restricted.

Figure 5 shows the calculated energy bands at selected lines of high symmetry in the Brillouin zone (see Fig. 3). At points at $\Delta$ and $\Sigma$ some extra $\vec{k}$ values were included in the calculation. At $\Delta$ these extra points (not marked on the figure) were concentrated at the crossings between bands 3 and 4 and between bands 5 and 6 . These degeneracies exist only when $\vec{k}$ is at a $\Delta$ line. The figure showing the bands at general points (Fig. 6) clearly demonstrates these splittings. In the subsequent discussion we always number the band curves from below in energy. Only at the two crossings at $\Delta$ mentioned above will there then be an artificial discontinuity in the group velocity $\nabla_{\overrightarrow{\mathbf{k}}} E(\overrightarrow{\mathrm{k}})$.

As anticipated in the first part of this section, the relativistic effects are large. The bottom of the band $\left(\Gamma_{6}^{+}\right)$is lowered by 0.230 Ry relative to the nonrelativistic value $\left[E\left(\Gamma_{1}\right)=0.0665 \mathrm{Ry}, E\left(\Gamma_{6}^{+}\right)\right.$ $=-0.1641 \mathrm{Ry}]$. Thus, the lowering is even larger than estimated from the atomic calculation ( $0.15 \mathrm{Ry})$ This is also what one might expect since the wave function in the solid (for $r<R_{s}$ ) is larger than the atomic wave function. The spin-orbit splitting of $\Gamma_{25}^{\prime}$ into $\Gamma_{8}^{+}$and $\Gamma_{7}^{+}$is $0.077 \mathrm{Ry}$, which agrees with the estimate $(0.08 \mathrm{Ry})$. But at $X\left(X_{5}-X_{6}^{+}+X_{l}^{+}\right)$the band calculation $\left(\Delta E_{\mathrm{so}}=0.078 \mathrm{Ry}\right)$ shows that the calculation $\left(\Delta E_{\mathrm{so}}=0.05 \mathrm{Ry}\right)$ using the atomic spinorbit parameter underestimates the splitting. Since $E\left(X_{6}^{+}\right)$and $E\left(X_{7}^{+}\right)$are larger than the levels $E\left(\Gamma_{7}^{+}\right)$ and $E\left(\Gamma_{8}^{+}\right)$considered, this shows that the spinorbit parameter $\frac{1}{2} \xi_{d}(E)$ increases with increasing energy $E$. The calculation of $\xi_{d}(E)$ for Pd in Ref. 20 from the functions $\alpha_{l}(E)$ and $\beta_{l}(E)$ also shows a 


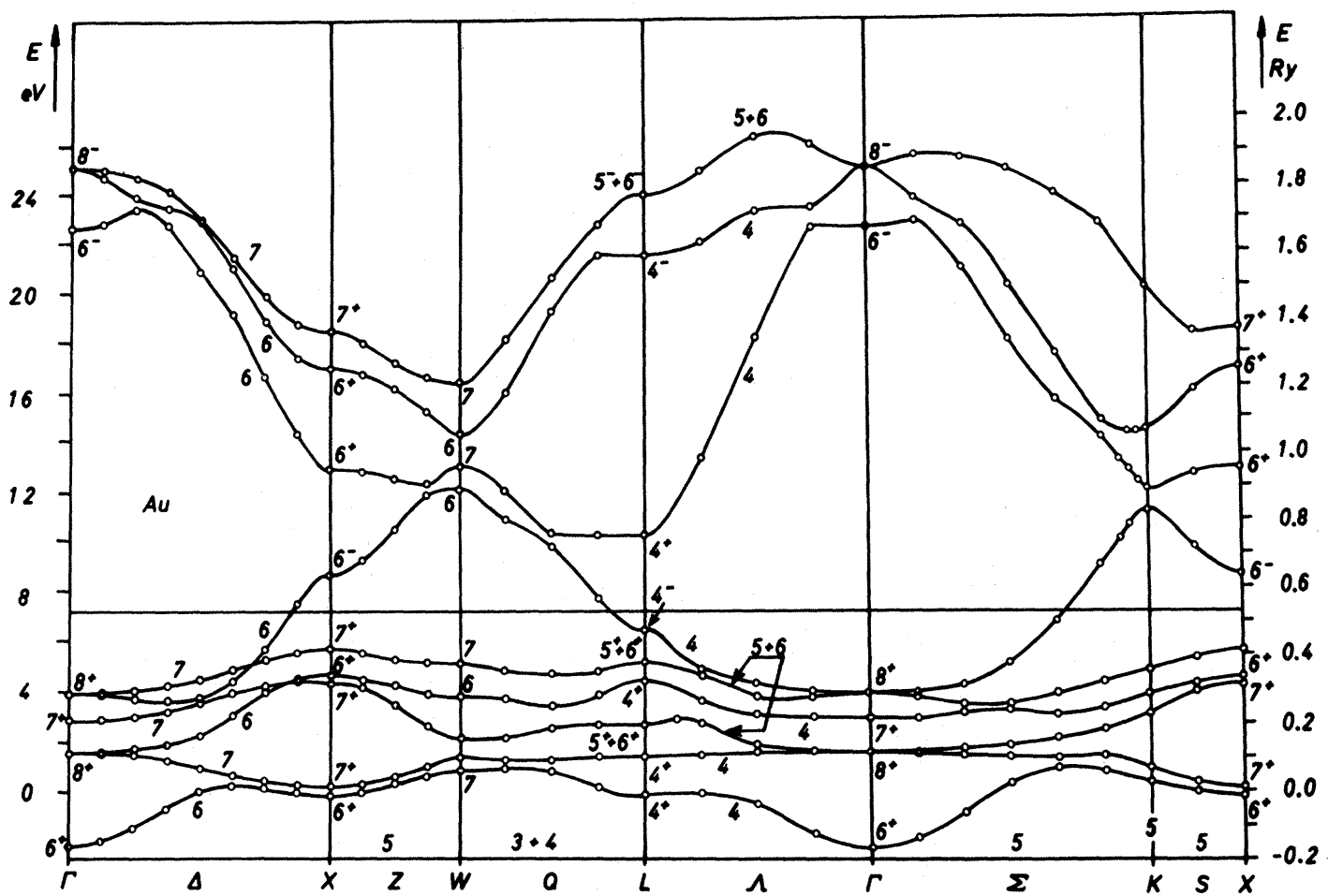

FIG. 5. RAPW energy bands of gold (normal volume). $E_{F}=0.530$ Ry. Energies are measured from the muffin-tin zero.

similar dependence.

The nonrelativistic calculation yielded 0.501 Ry for the $L_{2}^{\prime} \rightarrow L_{1}^{u}$ separation whereas the RAPW value is only $0.275 \mathrm{Ry}$. Thus inclusion of relativistic effects leads to a reduction of this transition energy by $0.226 \mathrm{Ry}$ or $3.05 \mathrm{eV}$. The particularly large effect at this gap is supposed to be caused partly by the $d$ states influencing the gap by means of the hybridization.

During the preparation of this paper, four other relativistic band calculations for gold have been reported. $3-26$ These are compared with our results in Table III. The $d$ bandwidth as determined by Sommers and $\mathrm{Amar}^{23}$ seems to be far too large

TABLE III. Band separations in gold. Relativistic calculations. Column a is from Sommers and Amar (Ref. 23) (2/3 Slater exchange); b, Conolly and Johnson (Ref. 25) (1/1 Slater exchange); c, Kupratakuln (Ref. 24) (19/24 Slater exchange); d, Ramchandani (Ref. 26) (1/1 Slater exchange).

\begin{tabular}{|c|c|c|c|c|c|c|}
\hline & $\mathrm{a}$ & $\mathrm{b}$ & $\mathrm{c}$ & d & $\begin{array}{c}\text { Present } \\
\text { RAPW } \\
\mathrm{e} \\
\end{array}$ & $\begin{array}{c}\text { Present } \\
\text { expt. lattice } \\
\end{array}$ \\
\hline \multicolumn{7}{|l|}{$s$ bandwidth } \\
\hline$X_{6}-\Gamma_{6}^{+}$ & 0.81 & 0.79 & 0,805 & 0.802 & 0.803 & 0.781 \\
\hline$X_{6}^{-}-L_{4}^{-}$ & 0.17 & 0.17 & 0.175 & 0.108 & 0.161 & 0.156 \\
\hline$L_{\overline{4}}^{-}-\Gamma_{6}^{+}$ & 0.64 & 0.63 & 0.630 & 0.594 & 0.641 & 0.625 \\
\hline \multicolumn{7}{|l|}{$d$ bandwidth } \\
\hline$X_{7}^{+}-X_{6}^{+}$ & 0.52 & 0.415 & 0.435 & 0.459 & 0.431 & 0.406 \\
\hline$\Gamma_{8}^{+}-\Gamma_{8}^{*}$ & 0.19 & 0.18 & 0.174 & 0.166 & 0.173 & 0.174 \\
\hline$L_{6+5}^{+}-L_{4}^{+}$ & 0.51 & 0.375 & 0.391 & 0.427 & 0.386 & 0.364 \\
\hline \multicolumn{7}{|l|}{$s-d$ separation } \\
\hline$L_{\overline{4}}-L_{6+5}^{+}$ & -0.11 & 0.115 & 0.074 & 0.096 & 0.101 & 0.112 \\
\hline$X_{6}^{-}-X_{7}^{+}$ & 0.05 & 0.25 & 0.207 & 0.176 & 0.221 & 0.229 \\
\hline \multicolumn{7}{|l|}{ Lattice const. } \\
\hline a.u. & & 7.706 & & 7.6799 & 7.6813 & 7.7820 \\
\hline
\end{tabular}



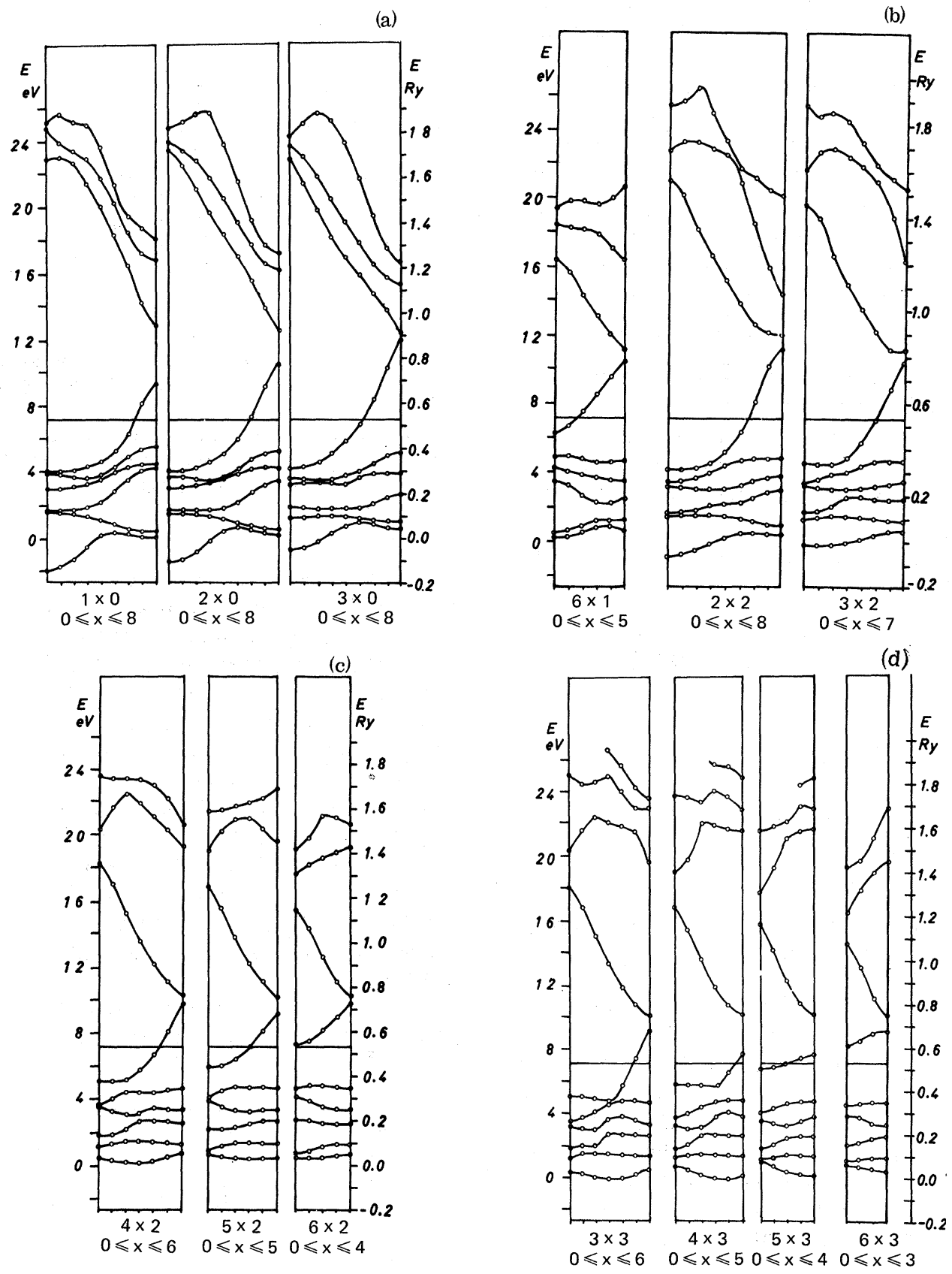

FIG. 6. RAPW energy bands of gold (normal volume), general points; $\vec{k}$ is in units of $\pi / 4 a$.

(Sec. V). They constructed their potential in a way similar to ours but included only $\frac{2}{3}$ exchange. In view of the discussion in Sec. II, it is clear that this must lead to bands that are broader than those we calculate with full Slater exchange. Sommers and Amar find a band crossover at $\Lambda$ whereas the corresponding bands $\Lambda_{4}$ and $\Lambda_{5+6}$ in our calculation are separated at all $\Lambda$ points. Further, their calculation near $K$ is not sufficiently detailed to rule out the existence of critical points in the joint den- sity-of-states function. That there is a possibility for such critical points can be seen from Fig. 5, and after a more detailed examination we actually found one, although not exactly the one discussed in Ref. 23. Our critical point corresponds to transitions from $\Sigma_{5}^{5}$ to $\Sigma_{5}^{8}$ very near $K$. The potentials applied in the calculations (c) and (d) in Table III are similar to the one we constructed. Probably the only differences consist of different $\Delta V_{0}$. It is therefore somewhat disappointing that there is no 
better agreement among the three calculations (c)(e). However, in (d) the RAPW expansion probably has not converged since only a small matrix was used. The energies of (d) must contain errors of at least $0.02 \mathrm{Ry}$ since there is that much fluctuations in the results at points that, due to symmetry, are equivalent. In (d), only 12 basis vectors were included, and in view of our convergence test (Fig. 4) it is obvious that this does not yield satisfactory accuracy for our calculation. This somewhat reduces the inconsistency of the columns (c)-(e).

C. Density of States and Fermi Level: Interpolation

Because the number of points $\vec{k}$ at which the energy values were calculated by the RAPW method is very small (89 in $\frac{1}{48}$ zone), it was necessary to interpolate the energy bands by some means in order to calculate the density-of-states functions

$N(E)=\frac{2 \Omega}{(2 \pi)^{3}} \int \frac{d S_{E}}{\left|\nabla_{\overrightarrow{\mathbf{k}}}[E(\overrightarrow{\mathrm{k}})]\right|}$

and

$$
\begin{aligned}
J(\hbar \omega)= & \frac{2 \Omega}{(2 \pi)^{3}} \sum_{i, j} \int d^{3} k f\left(E_{i}(\overrightarrow{\mathrm{k}})\right)\left[1-f\left(E_{j}(\overrightarrow{\mathrm{k}})\right)\right] \\
& \times \delta\left(E_{j}(\overrightarrow{\mathrm{k}})-E_{i}(\overrightarrow{\mathrm{k}})-\hbar \omega\right) \\
= & \frac{2 \Omega}{(2 \pi)^{3}} \sum_{\substack{i, j \\
E_{i}<E_{F} \\
E_{j}>E_{F}}} \int \frac{d S_{h \omega}}{\left|\nabla_{\overrightarrow{\mathrm{k}}}\left[E_{i}(\overrightarrow{\mathrm{k}})-E_{j}(\overrightarrow{\mathrm{k}})\right]\right|},
\end{aligned}
$$

$N(E)$ being the one-particle density of states and $J(\hbar \omega)$ the joint density of states.

Three methods of interpolation were tried. A combined OPW-LCAO scheme similar to the one suggested by Ehrenreich and Hodges ${ }^{27}$-with spinorbit coupling-was fitted to the initial RAPW bands. It was, however, impossible to achieve rms errors less than $19 \mathrm{mRy}$. Therefore it was decided to consider purely mathematical interpolation methods. A global interpolation based on a least-squaresfitted series of symmetrized plane waves (SPW) led to small rms errors (Table IV). Band 6 is not

TABLE IV. rms errors in interpolation based on a series of symmetrized plane waves as a function of the number of SPW's included in the expansion. The errors are in units of $0.001 \mathrm{Ry}$.

\begin{tabular}{ccccccc}
\hline \hline No. & Band & Band & Band & Band & Band & Band \\
of SPW's & 1 & 2 & 3 & 4 & 5 & 6 \\
\hline 13 & 3.1 & 3.5 & 7.5 & 7.5 & 5.0 & \\
20 & 1.6 & 1.8 & 2.7 & 3.1 & 3.2 & 10.4 \\
30 & 0.85 & 1.1 & 2.4 & 1.9 & 2.2 & 7.6 \\
40 & 0.65 & 0.85 & 1.6 & 1.5 & 1.5 & \\
49 & 0.6 & 0.7 & 1.4 & 1.3 & 1.4 & 4.5 \\
\hline \hline
\end{tabular}

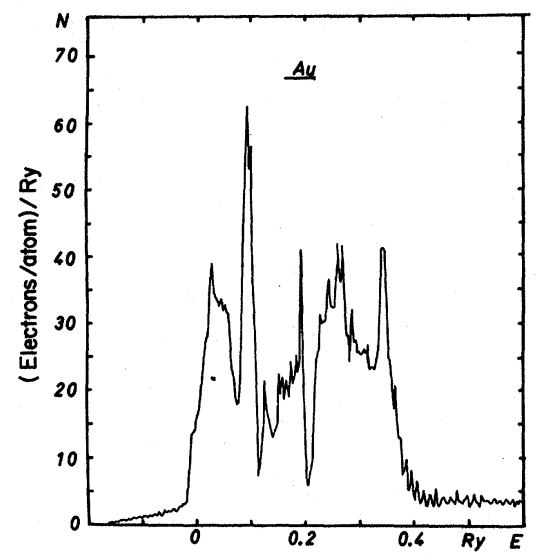

FIG. 7. Density-of-states function. Histogram calculation based on SPW expanded bands. $7216 \overrightarrow{\mathrm{k}}$ in $\frac{1}{48}$ zone. $49 \mathrm{SPW}$ 's were used in the expansion.

flat as are the five lower ones, and it is therefore more difficult to approximate by the SPW series. Probably better results for this band would have been obtained if cubic harmonics had been used instead of SPW's. The density-of-states function (2) was calculated as a histogram, i. e., by scanning a small energy window along the energy scale and at intervals $\Delta E$ counting the number of energies in the interval $E_{ \pm} \Delta E / 2$, each attributed its proper weight. For this purpose, 49 SPW's were included in the series and $N(E)$ corresponding to several sets of points $\vec{k}$. Figure 7 shows the result obtained with $7216 \overrightarrow{\mathrm{k}}$ 's in $\frac{1}{48}$ zone. Even when that many points are included, the statistical fluctuations are quite large.

The most successful way of calculating functions (2) and (3) seemed to be a combination of local

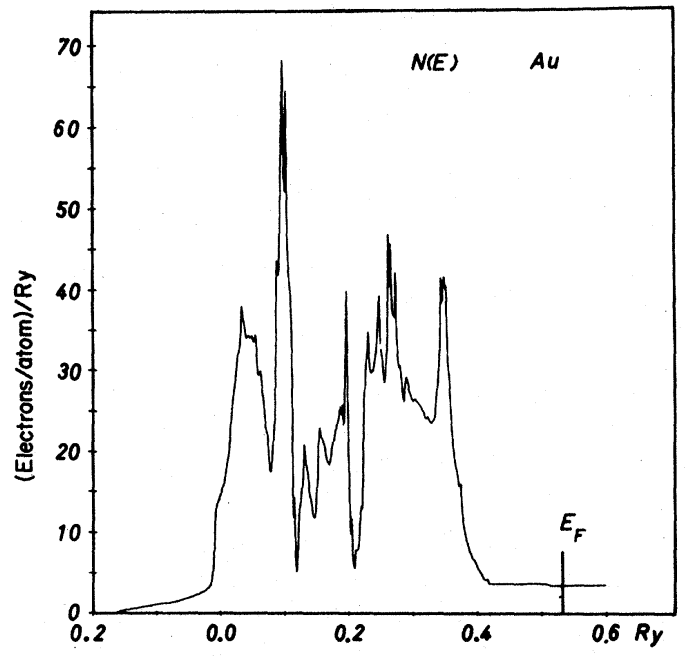

FIG. 8. Density-of-states of gold. Gilat-Raubenheimer calculation. 
Lagrange interpolation and the method suggested by Gilat and Raubenheimer. ${ }^{28}$

Figure 8 shows $N(E)$ for gold calculated by this scheme. Now there are no statistical oscillations on the $s p$ parabola, and the density of states at the Fermi level agrees with an accurate calculation of constant-energy surfaces (CES) calculated by the RAPW method programmed for $\overrightarrow{\mathrm{k}}$ search. ${ }^{29}$ The CES integrations as well as the calculation of $N\left(E_{F}\right)$ yields a thermal effective-mass ratio $m_{t} / m_{0}=1.01$ \pm 0.01 . The experimental value obtained by $\operatorname{Martin}^{30}$ is 1.09 , indicating an average apparent ${ }^{31}$ electronphonon enhancement of $8 \%$. Also the joint densityof-states function $J(\hbar \omega)$ was evaluated by the GilatRaubenheimer method. Figures 9-11 show the partial joint density-of-states functions $J_{i j}(\hbar \omega)$, i. e. , the densities of transitions of energy $\hbar \omega$ from the part of band $i$ that is below the Fermi level to the part of band $j$ that is above $E_{F}$. The total joint density of states is the sum of all $J_{i j}(\hbar \omega)$. This function is discussed later (Figs. 19-21).

The Fermi level $E_{F}$ was calculated by CES integrations. The $d$ bands are known to be filled, and thus the volume $V_{F S}$ of the Fermi surface must equal one-half the volume of the first Brillouin zone.

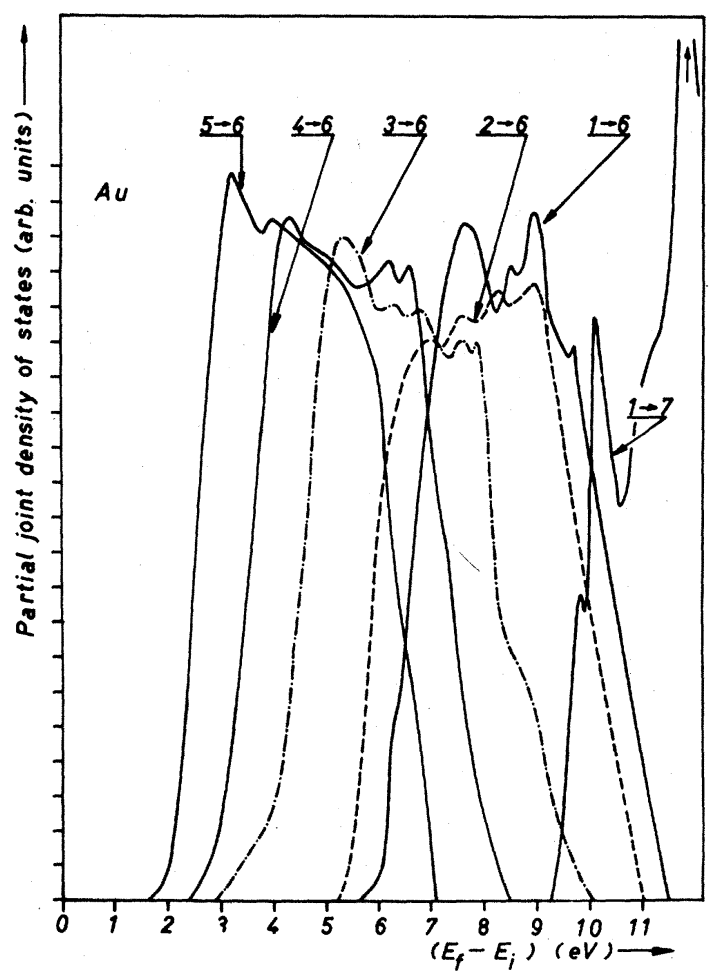

FIG. 9. Partial joint density-of-states functions (GilatRaubenheimer calculation). Number of transitions from band $i$ to band $f$ as a function of photon energy, $\hbar \omega=E_{f}$ $-E_{i}$. Only transitions for which $E_{f}>E_{F}$ and $E_{i}<E_{F}$ are included; $(i, f)=(1,6),(1,7),(2,6),(3,6),(4,6)$, and $(5,6)$.

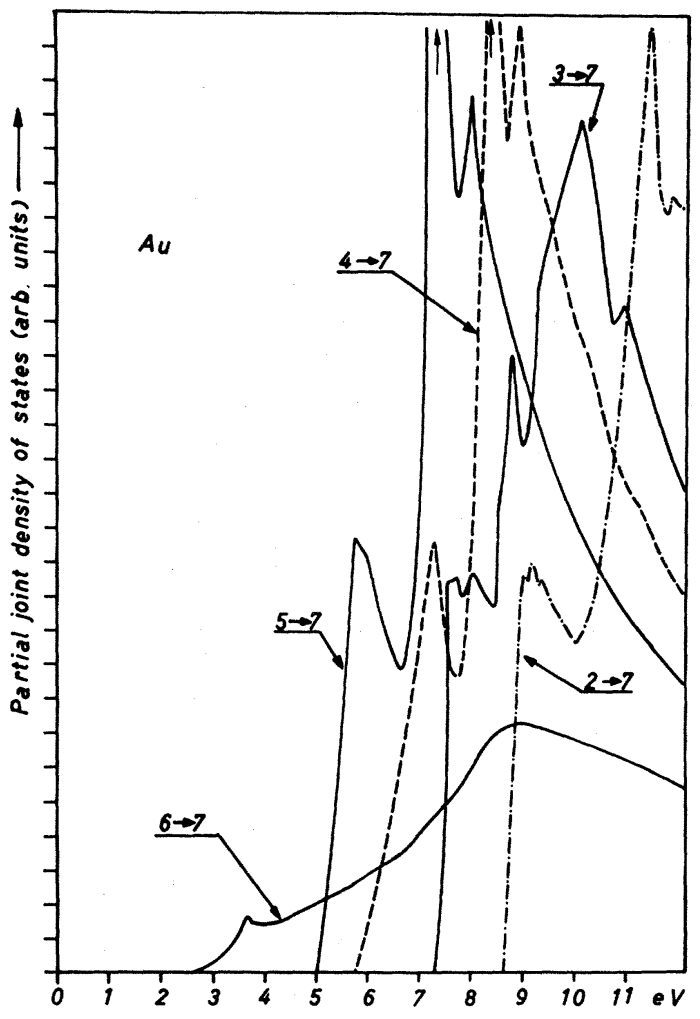

FIG. 10. Similar to Fig. 9 but $(i, f)=(2,7),(3,7),(4,7)$, $(5,7)$, and $(6,7)$.

Three CES's were calculated by the RAPW method. The Fermi level was in this way determined to be $E_{F}=0.530 \mathrm{Ry}$, since the CES corresponding to this energy has the volume $1.000 V_{\mathrm{FS}}$. It is not unusual to see calculations of $E_{F}$ performed by integrating histogram density-of-states functions obtained from as few as 89 points in $\frac{1}{48}$ zone, and results quoted with three or four decimal places. We tried to calculate $E_{F}$ in this way and found $E_{F}=0.51 \mathrm{Ry}$, indicating large errors in the second decimal place when compared to the more accurate value. In Fig. 12 are plotted the integrals

$$
I(E)=\int_{E_{0}}^{E} N\left(E^{\prime}\right) d E^{\prime} \quad\left(E_{0}=-0.2 \mathrm{Ry}\right)
$$

vs $E$ for different density-of-states functions. $E_{F}$ is the value of $E$ making $I(E)=11 . E_{F 1}, E_{F 2}$, and $E_{F 4}$ correspond to $N(E)$ functions calculated as histograms using SPW expansion of the bands. Only when a very large number of $\vec{k}$ 's are used-several thousand in $\frac{1}{48}$ zone-can $E_{F}$ be reasonably well determined by integration of the histogram. Further, when the Gilat-Raubenheimer-calculated density of states is integrated it gives a Fermi level $\left(E_{F 3}\right)$ that agrees with the CES result. 


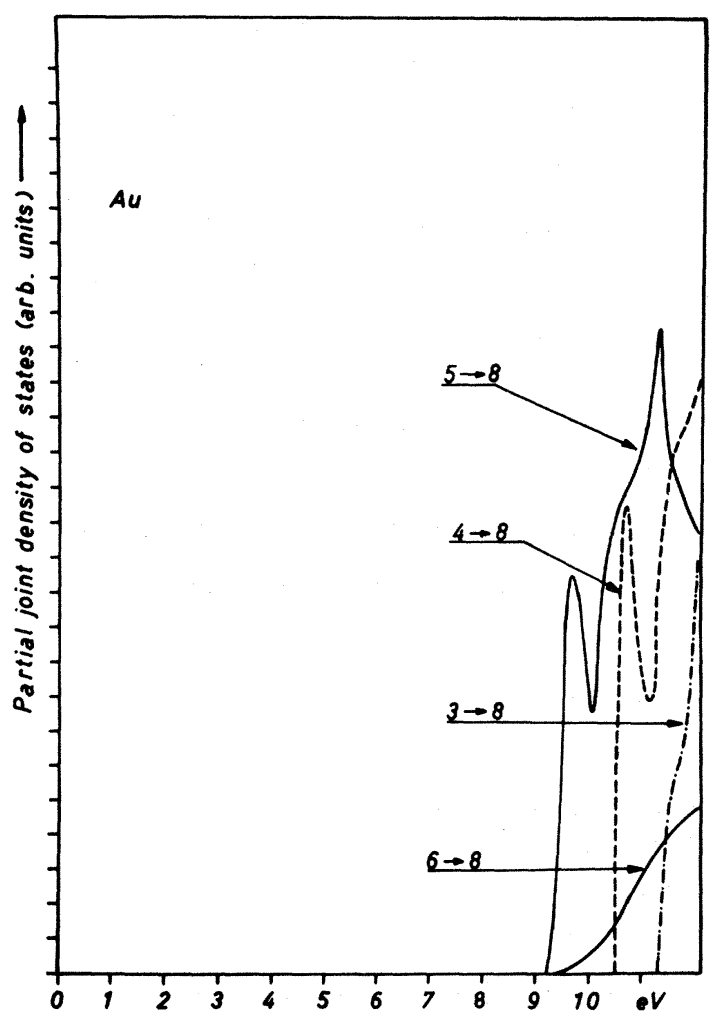

FIG. 11. As Figs. 9 and 10 but $(i, f)=(3,8),(4,8),(5,8)$, and $(6,8)$.

\section{Expanded Lattice: Strain Responses}

Expansion of the lattice causes free-electronlike bands to move toward lower energies as they behave more or less like $k^{2}$. Simultanously, the $d$ bands become narrower owing to the reduction of the potential overlap. When the distance between the atomic sites increases, the centrifugal barrier becomes more effective in the sense that the probability for a $d$ electron to tunnel through it decreases. Thus the lifetime of the virtually bound state is increased and the bandwidth is reduced. Further, measured relative to the actual muffintin zero $V_{0}$, the center of gravity of the $d$ bands will move to lower energies when the lattice is expanded.

The RAPW calculation for the expanded Au lattice covers all 89 points. However, only eigenvalues at some symmetry points are included here (Table V). Band separations are compared to the normal volume results in the last column of Table III.

The narrowing of the $d$ bands and the change of their center of gravity are so large at $X$ that the separation between the $d$ top and the $(s) p$ level $X_{6}^{-}$ increases although $X_{6}^{-}$moves to a lower energy. Further, as seen from Table V, the transition $L_{4}^{-} \rightarrow L_{4}^{+}$is strongly affected by strain (see also Fig. 18).

Considering the conduction band near $E_{F}$, it is not surprising that the states at the regions near $X$ and $L$ are the most sensitive to strain. These are just the regions where the Fermi surface exhibits the largest deviations from the spherical shape, indicating that electrons on the Fermi surface sense the crystal potential particularly strongly. Possibly this fact is also reflected in the large anisotropy in the electron-phonon renormalization. ${ }^{31}$

\section{E. Phonons: Debye-Waller Factors}

Simulating the temperature shifts in the optical spectra using our band model, we could not a priori expect a static expansion of the lattice to reproduce such temperature effects satisfactorily. The thermal vibrations will also influence the band structure-at least by a smearout of the crystal potential. ${ }^{32}$ An accurate calculation of the temperature responses is difficult owing to the $d$ bands. But an estimate of the order of magnitude of the shifts in the conduction states due to the vibrations can be obtained in a simple way.

Considering the $s p$ bands, an average smearout effect can be accounted for by multiplying the pseudopotential Fourier constants in an OPW-LCAO

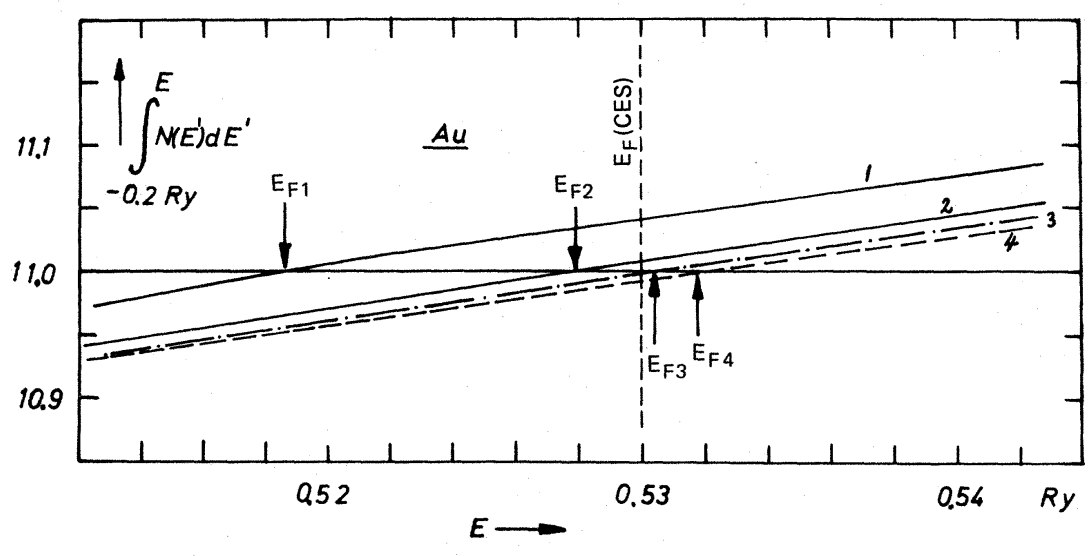

FIG. 12. Number of band-electron states per atom with energy less than $E$ as a function of $E$. Results based on different one-electron density-of-states functions and the corresponding Fermi levels: (1) SPW expansion, $2305 \vec{k}$ in $\frac{1}{48}$ zone; (2) $3346 \vec{k}$; (4) $7216 \vec{k}$; (3) is the integrated Gilat-Raubenheimer density of states. Vertical broken line gives the Fermi level obtained from RAPW constant-energy surfaces (Ref. 29). 
TABLE V. RAPW eigenvalues. Dilated lattice. The crystal volume is increased by $3.9 \%$ compared to the normal lattice. The energies are in Ry and measured relative to $V_{0}=-1.0317 \mathrm{Ry}$.

\begin{tabular}{cccccccccc}
\hline Band & 1 & 2 & 3 & 4 & 5 & 6 & 7 & 8 \\
\hline$\Gamma(000)$ & -0.1665 & 0.0949 & 0.0957 & 0.1943 & 0.2639 & 0.2654 & 1.6451 & 1.8051 \\
$\Delta(040)$ & -0.0090 & 0.0565 & 0.1429 & 0.2394 & 0.2551 & 0.3025 & 1.4943 & 1.6418 & 1.6564 \\
$X(080)$ & -0.0203 & 0.0062 & 0.2979 & 0.3087 & 0.3860 & 0.6145 & 0.9025 & 1.2176 & 1.3194 \\
$W(480)$ & 0.0420 & 0.0831 & 0.1413 & 0.2513 & 0.3441 & 0.8489 & 0.9236 & 1.0192 & 1.1637 \\
$K(660)$ & 0.0188 & 0.0574 & 0.2077 & 0.2576 & 0.3292 & 0.7878 & 0.8517 & 1.0224 & 1.4460 \\
$\Sigma(440)$ & 0.0581 & 0.0812 & 0.1418 & 0.2080 & 0.2653 & 0.4623 & 1.1081 & 1.2466 & 1.7264 \\
$Z(280)$ & 0.0069 & 0.0301 & 0.2317 & 0.2838 & 0.3552 & 0.7422 & 0.8766 & 1.1537 & 1.2265 \\
$L(444)$ & -0.0178 & 0.0852 & 0.1796 & 0.2946 & 0.3466 & 0.4585 & 0.7070 & 1.5607 & 1.7234 \\
\hline \hline
\end{tabular}

scheme by the appropriate Debye-Waller factors, $e^{-W} .{ }^{33}$ The Debye-Waller exponent corresponding to the reciprocal lattice vector $\vec{G}$ (in units of $2 \pi / a$ ) is $^{34}$

$$
W=\frac{1}{2} \frac{G^{2} \hbar}{N M} \frac{1}{8 \pi^{3}} \frac{1}{3} \sum_{p 01} \int \frac{\left\langle n_{q}\right\rangle+\frac{1}{2}}{\omega_{\mathbb{q}}} d^{3} q,
$$

where

$$
\left\langle n_{\overrightarrow{\mathrm{q}}}\right\rangle=\frac{1}{e^{\hbar \omega_{\mathbb{q}} / k T}-1}
$$

and $M$ is the ion mass.

Equations (5) and (6) show that we must know the phonon frequencies $\omega_{\mathbb{q}}$ and the spectrum $N(\omega)$-the density of phonon states.

The phonon dispersion relations were evaluated by using the simple model applied by Sharma and $\mathrm{Joshi}^{35}$ and by Gupta. ${ }^{36}$ In this model, the phonon dispersion relations are calculated from knowledge of only the elastic constants, the ionic mass, and the lattice geometry. Thus, the dispersive behavior of $\omega(\vec{q})$ is determined from knowledge about the nondispersive regime as obtained by measurements of sound velocities. In a metal, knowledge of the interactions between the ions and the electrons is essential for determining the phonon frequencies. This electron-ion interaction is accounted for by describing the conduction electrons as a uniform gas surrounding the ions and having a bulk modulus that has to be adjusted to the experimental sound velocities. In the present model, the forces between the ions are assumed to be central, and only nearest- and next-nearest-neighbor ion-ion interactions are included. Under these assumptions, and averaging the electronic effects over a WignerSeitz sphere, the equations of motion for the ions are deduced. These equations contain the ion-ion force constants and the electronic bulk modulus. Denoting by $\alpha$ the nearest-neighbor force constant, by $\beta$ the next-nearest neighbor constant, and by $B_{e}$ the electronic bulk modulus, the phonon frequencies $\omega_{\vec{q}}$ are obtained from $^{35,36}$

$$
\operatorname{det}\left\{\underline{\mathrm{D}}-M \omega_{\bar{q}}^{2} \underline{\mathrm{I}}\right\}=0,
$$

where $M$ is the ion mass, $\underline{I}$ is the unit matrix, and D is given by
$D_{i i}=4 \alpha\left(\cos \frac{1}{2} q_{i} a \cos \frac{1}{2} q_{j} a+\cos \frac{1}{2} q_{i} a \cos \frac{1}{2} q_{k} a-2\right)$

$$
-4 \beta \sin ^{2} \frac{1}{2} q_{i} a+\frac{1}{4} B_{e} a^{3} G^{2}\left(q R_{\mathrm{ws}}\right) q_{i}^{2}
$$

and

$D_{i j}=-4 \alpha \sin \frac{1}{2} q_{i} a \sin \frac{1}{2} q_{j} a+\frac{1}{4} B_{e} a^{3} G^{2}\left(q R_{\mathrm{ws}}\right) q_{i} q_{j}$.

In these expressions $a$ is the lattice constant, $R_{\mathrm{ws}}$ is the Wigner-Seitz sphere radius, and the function $G(x)$ is

$$
G(x)=3\left\{\sin x / x^{3}-\cos x / x^{2}\right\} .
$$

The parameters $\alpha, \beta$, and $B_{e}$ can be expressed in terms of the elastic constants by taking the longwavelength limit of Eqs. (7)-(9) and requiring them to yield the correct sound velocities in symmetry directions. The results are

$$
\begin{aligned}
\alpha & =-\frac{1}{2} a c_{44}, \\
\beta & =-\frac{1}{4} a\left(c_{11}-c_{12}-c_{44}\right), \\
B_{e} & =c_{12}-c_{44} .
\end{aligned}
$$

Inserting the elastic constants as derived from the experiments by Neighbours and Alers ${ }^{37}$ at zero temperature, we calculated the phonon frequencies at 10569 points $\vec{q}$ in $\frac{1}{48}$ zone and constructed the density-of-states function $N(\omega)$ as a histogram (Fig. 13). The points $\vec{q}$ were distributed in a uniform mesh, and to each point was attributed the proper weight according to the symmetry. In the rather crude model that we use, we always apply the same elastic constants although in reality they vary with the temperature $T$. In evaluating $W(T)$, we, in fact, ought to recalculate $N(\omega)$ at each temperature using the corresponding elastic constants. The DebyeWaller exponent for a unit reciprocal lattice vector as a function of temperature $T$ is shown in Fig. 14.

Although the rms error in the fit of the OPW-

LCAO scheme (with spin-orbit coupling) was 19 mRy, we used this scheme in estimating the effects on the band structure due to the lattice vibrations. The order of magnitude of the shifts due to the potential smearout was obtained for the conduction states by multiplying the pseudopotential Fourier 


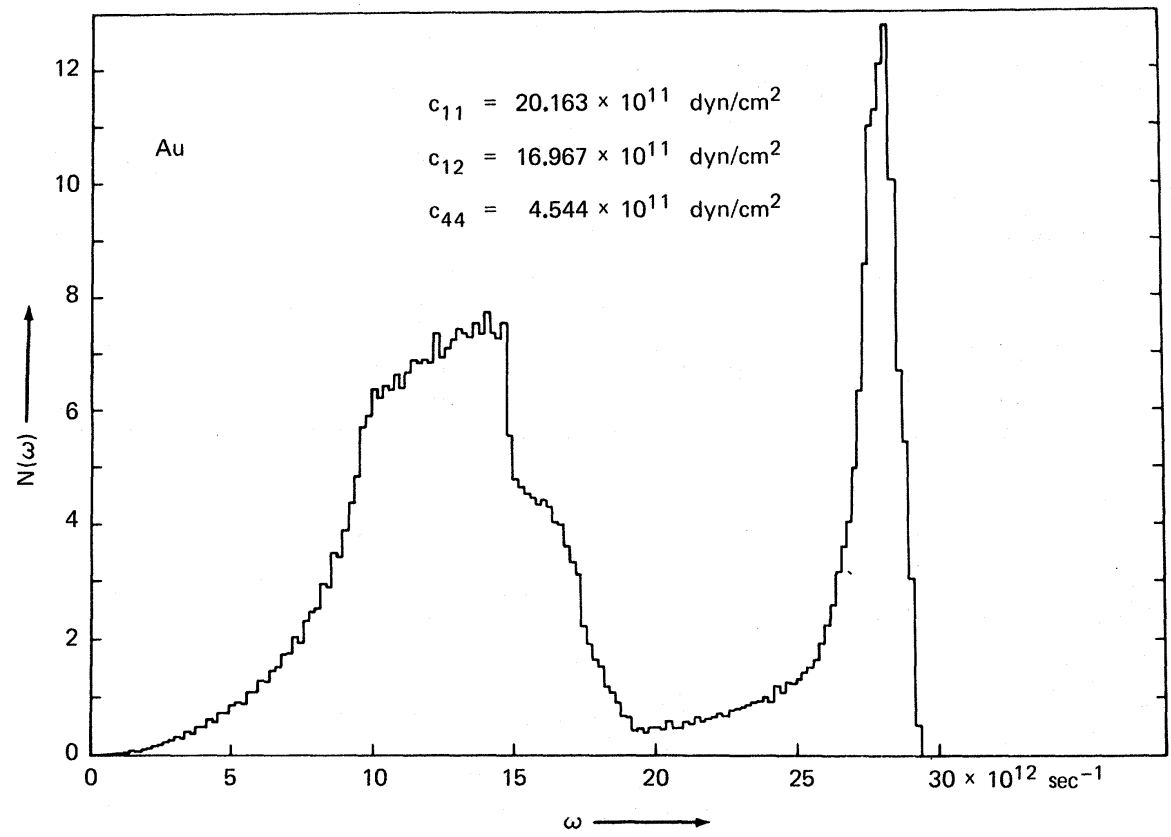

FIG. 13. Phonon spectrum of gold calculated as a histogram based on frequencies at 10569 $\overrightarrow{\mathrm{q}}$ vectors in $\frac{1}{48}$ zone. Characteristic data for the spectrum: $\omega_{\max }=29.4 \times 10^{12} \mathrm{sec}^{-1}=224.6 \mathrm{~K}$, $\langle\omega\rangle / \omega_{\max }=0.573,\left\langle\omega^{2}\right\rangle / \omega_{\max }^{2}$ $=0.391,[\langle\omega\rangle /\langle 1 / \omega\rangle] / \omega_{\max }^{2}$ $=0.264$.

constants $V(\mathbb{G})$ by $e^{-w}$. The largest shift was observed at $X_{6}^{-}$, where it was $0.002 \mathrm{Ry}$ or $27 \mathrm{meV}$ if $T$ is changed from 0 to $750 \mathrm{~K}$. Since this is small compared to the strain response, we conclude that the calculated strain responses really give the temperature shifts in the band structure.

\section{OPTICAL EXPERIMENTS: INTERPRETATION RULES}

The theoretical model of the electronic structure and the numerical results have been described in Secs. I-III. We want to check this against experimental evidence, and therefore it is necessary to discuss how connections between the band calculation and the optical experiments can be established. We will discuss the possible existence of effects from localization of the $d$ electrons, and in this

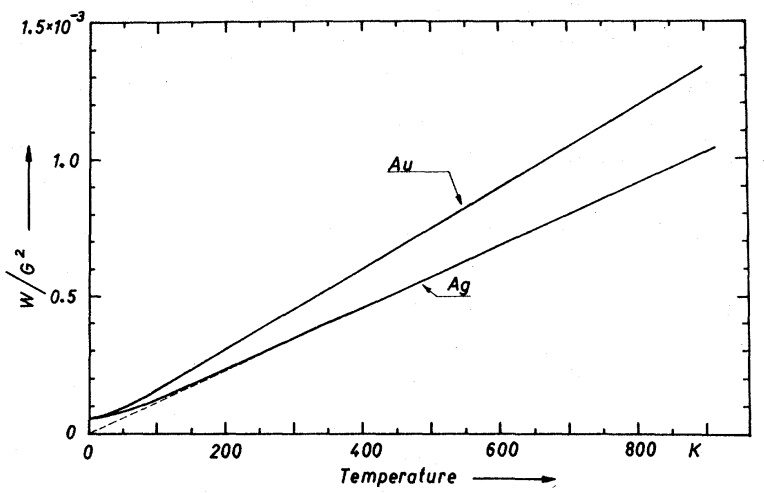

FIG. 14. Debye-Waller exponents $W$ for a unit $(2 \pi / a)$ reciprocal lattice vector $\overrightarrow{\mathrm{G}}$ as a function of temperature $T$. (Silver results included for comparison.) context study the importance of nondirect transitions.

\section{A. Localization of $d$ States}

In relating the calculated energy differences to observed excitation energies, we must apply an analog to Koopmans's theorem. The derivation of Koopmans's theorem is based on two assumptions: (i) that the Hartree-Fock approximation is valid, and (ii) that all one-electron orbitals are unchanged under the excitation. The latter assumption will not hold if the states involved in the excitation are localized. The states of the electrons within a screening length from the localized hole formed by the excitation will have to be described in terms of a completely new Hamiltonian, and the wave functions will be changed. Therefore, even if we had used the Hartree-Fock method in the band calculation, there might be effects from localization of the $d$ states, and only if by some means we could assert that the $d$ bands are wide enough to be considered as representing good band states could we apply Koopmans's theorem.

Now, condition (i) is not fulfilled. We do not apply the Hartee-Fock scheme, but a scheme with the simpler statistical exchange term. This implies that the eigenvalues we calculate $\left(\epsilon_{i}\right)$ do not correspond with discrete changes in the occupation number. In the Hartee-Fock scheme the one-particle energies are $\epsilon_{i}=\Delta H_{n_{i} \rightarrow n_{i}-1}$, the change in the total energy when the occupation number $n_{i}$ of the orbital $i$ is changed by 1 [assuming that condition (ii) is fulfilled]. When we use the statistical exchange, then $\epsilon_{i}=\partial H / \partial n_{i}$, the derivative of the total 
energy with respect to occupation numbers. ${ }^{38}$ Only in cases where the bands are wide is the $\epsilon_{i}$ calculated in this way the term that we want to compute because only then are the excitations extended rather than localized.

The crystal wave function consists of Bloch waves, and the energy needed to remove an electron is $H\left(n_{i}\right)-H\left(n_{i}-1\right), n_{i}$ being the number of electrons in Bloch state $i$. This is equal to $\partial H / \partial n_{i}$ $-\frac{1}{2}(i, i),(i, i)$ being the self-energy. ${ }^{38}$ Only when the state $i$ is widely spread throughout the crystal is $(i, i)$ vanishingly small, and the calculated quantity $\partial H / \partial n_{i}$ is the energy needed to move an electron from the crystal. Still, in deriving $\epsilon_{i}=\partial H / \partial n_{i}$, it is assumed that the wave functions are unchanged during the excitation. But this assumption is more reasonable the wider the bands are.

So, only if the $d$ states are good band states can we compare the calculated band structure to the results of optical experiments. The crucial point is then to make a distinction between localized and extended states.

Photoemission experiments give a hint about the importance of localization effects. The degree of localization of the $d$ states will be reflected in the photon-energy responses in the distribution of emitted electrons since the type of transition-direct or nondirect-is related to localization effects. ${ }^{4,5}$ If the $d$ states are localized, there will be possibilities for nondirect transitions. These are not vertical in $\overrightarrow{\mathrm{k}}$ space, but $\overrightarrow{\mathrm{k}}$ conservation is provided by momentum exchange with the localized holes. If there are no localized holes, as in the case of wide bands, such an exchange is not possible and the transitions are direct.

The energy distribution curves (EDC) obtained from the photoemission experiment will in cases where the transitions are exclusively nondirect reflect ${ }^{3}$ the product of the densities of initial and final states. In the noble metals, the final states are free-electron-like and the density-of-states function for these states is fairly smooth. Therefore, considering an EDC plotted-vs-initial state energy, it should be possible to extract the $d$ hump of the density of states. At least the spectral positions of peaks in the two curves should coincide. The amplitudes will differ by variations of the escape probability ${ }^{3}$ and matrix elements.

In a model assuming all transitions to be direct we must compare the EDC's to a function $D\left(E_{i}, \hbar \omega\right)$ that may be quite different from the density - of states product. $D\left(E_{i}, \hbar \omega\right)$ is the energy distribution of joint density of states. It gives the number of vertical transitions with a given initial energy $E_{i}$ and transition energy $\hbar \omega$. The function $D\left(E_{i}, \hbar \omega\right)$ is given by

$$
D\left(E_{i}, \hbar \omega\right)=C \sum_{n, m} \int d^{3} k f\left(E_{n}(\overrightarrow{\mathrm{k}})\right)\left[1-f\left(E_{m}(\overrightarrow{\mathrm{k}})\right)\right]
$$

$$
\times \delta\left(E_{n}(\overrightarrow{\mathrm{k}})-E_{i}\right) \delta\left(E_{m}(\overrightarrow{\mathrm{k}})-E_{n}(\overrightarrow{\mathrm{k}})-\hbar \omega\right) .
$$

The function $D\left(E_{i}, \hbar \omega\right)$ of Eq. (12) has been examined in detail by Koyama and $\mathrm{Smith}^{39}$ in their discussion of nondirect and direct models for photoemission properties of simple metals.

If the EDC's are plotted versus the initial state energy, then nondirect transitions from points $E_{i}(\overrightarrow{\mathrm{k}})$, where the density of states is large, will manifest themselves in peaks in the EDC's that remain stationary during variation of the photon energy. In general, peaks corresponding to direct transitions will move on the $E_{i}$ scale when EDC's with different $\hbar \omega$ are compared.

However, in actual cases it is not easy to distinguish between the two types of transitions. If the initial bands are flat, then even the peaks corresponding to direct transitions will remain stationary when the photon energy is changed. Further, a large level broadening can imply that $D\left(E_{i}, \hbar \omega\right)$ becomes similar to the product of the initial and final density of states. In the photoemission experiments, it is customary to cover the sample surface with a thin layer of cesium in order to lower the work function. Part of this Cs layer will diffuse into the sample metal and may form impurities of concentration and concentration gradients large enough to produce considerable changes of the band structure in the part of the sample reached by the photons. ${ }^{40}$ In fact, it cannot be excluded that these changes are such that when the photon energy, and thus the escape depth, is varied, then a peak corresponding to nondirect transitions responds similarly to a peak of direct transitions. Thus, if the EDC peaks there is a possibility that nondirect transitions are important, and we must be careful since this may imply that localization effects are not negligible. On the other hand, if the peaks do move, then a very detailed comparison between experiments and a theoretical model assuming direct transitions is necessary (Eq. 12). ${ }^{41}$ If such comparison shows full agreement, it is reasonabe to interpret further optical experiments in terms of direct transitions and to do this on the basis of the calculated band structure, i. e., assuming the $d$ states to be band states.

\section{B. Static and Modulated Reflectance}

The complex dielectric constant $\epsilon(\omega)$ has two contributions, $\epsilon^{(c)}(\omega)$ and $\epsilon^{(i)}(\omega)$, the intraband and interband terms, respectively:

$$
\begin{aligned}
\epsilon(\omega)=\epsilon_{1}(\omega)+i \epsilon_{2}(\omega)=[ & \left.\epsilon_{1}^{(c)}(\omega)+\epsilon_{1}^{(i)}(\omega)\right] \\
& +i\left[\epsilon_{2}^{(c)}(\omega)+\epsilon_{2}^{(i)}(\omega)\right] .
\end{aligned}
$$

Assuming that the intraband contributions to the dielectric function can be subtracted in the analysis of the experimental results, we will concentrate 
on the interband part of $\epsilon_{2}(\omega)$ since this parameter relates more directly to the band structure.

The static reflectance and transmittance experiments measure the total absorption at a given photon energy $\hbar \omega$. The sum total of all direct interband transitions at this energy is proportional to $\epsilon_{2}^{(i)}(\hbar \omega)$ :

$$
\begin{array}{r}
\epsilon_{2}^{(i)}(\omega)=C \sum_{i, f} \int\left|M_{i f}\right|^{2} f\left(E_{i}(\overrightarrow{\mathrm{k}})\right)\left[1-f\left(E_{f}(\overrightarrow{\mathrm{k}})\right)\right] \\
\times \delta\left(E_{f}(\overrightarrow{\mathrm{k}})-E_{i}(\overrightarrow{\mathrm{k}})-\hbar \omega\right) d^{3} k,
\end{array}
$$

where $C$ is a constant, $f(E)$ is the Fermi function, and $M_{i f}$ is the matrix element for transition from state $i$ to state $f$. If the transition matrix elements were constant, this would imply

$$
\epsilon_{2}^{(i)}(\omega) \omega^{2} \propto J(\hbar \omega),
$$

where $J(\hbar \omega)$ is the joint density-of-states function discussed earlier [Eq. (3)]. At critical points, points in $\overrightarrow{\mathrm{k}}$ space where $\nabla_{\overrightarrow{\mathrm{k}}}\left[E_{i}(\overrightarrow{\mathrm{k}})\right]=\underline{0}$, the integrand in (3) will contain a singularity. Its potential to generate significant structure in the spectral profile of $\epsilon_{2}$ has in the past been frequently overemphasized. Consequently, such structure was assigned to band gaps at specific critical points. As pointed out in particular by Herman et al. , ${ }^{8}$ such practice can lead to serious and consequential errors if the assignment is used for an adjustment of the crystal potential.

Although undoubtedly present in the experimental trace, the resolution of static reflectance techniques is too low to resolve the contribution of the criticalpoint singularity from the large background originating in extended, noncritical regions of the Brillouin zone. The spectral profile of the static trace reflects that of the integral of Eq. (14) rather than the line shape of a localized contribution.

Summing up, we can state that critical points do generate structure in the optical spectrum $\epsilon_{2}(\omega)$, but not necessarily all structure results from critical points. Their contribution probably appears as fine structure only, which may or may not be near the broad peaks with which they must not be exclusively associated. ${ }^{8,42}$ Further, the structure in $\epsilon_{2}(\omega)$ due to critical points would only in rare cases be expected to be shaped like a peak, as this would call for the unlikely arrangement of two critical points back-to-back at nearly the same energy.

To get experimental information about the transition energies at localized points in $\vec{k}$ space, we must turn our attention to the modulated reflectance measurements. The separation of the criticalpoint transitions from the background is probably accomplished by utilizing the analytic singularities of the integrand in (3). Near a critical point, $J_{i f}(\hbar \omega)$ is parabolic. If we assume that the modulation response $\Delta \epsilon_{2}$ can be derived from (15) with the modulation resulting in changes $\Delta E_{g}$ in the spectral posi- tion $E_{g}$ of the gap, then

$$
\frac{\Delta \epsilon_{2}}{\Delta E_{g}}=\frac{C}{\omega^{2}} \frac{\Delta J}{\Delta E_{g}}
$$

As an example of a critical point, let us consider one of type $M_{3}$, i. e. , $J_{i f}(\hbar \omega)=C_{1}+C_{2}\left(E_{g}-\hbar \omega\right)^{1 / 2}$. Then $\Delta J / \Delta E_{g}$ behaves like

$$
\frac{d J_{i f}}{d E_{g}}=\frac{C_{3}}{\left(E_{g}-\hbar \omega\right)^{1 / 2}} .
$$

The response disappears as the spectral distance from the critical point increases. Noncritical areas in which the joint density-of-states function is smooth and free of square-root slope discontinuities should not be affected by the modulation. ${ }^{42}$

The $\Delta \epsilon_{2}$ spectrum is derived by Kramers-Kronig analysis from an experimental $\Delta R / R$ trace. It depends on the particular choice of the modulation parameter. We will mostly be concerned with thermoreflectance. In addition to the criticalpoint responses, this spectrum will contain structure elements corresponding to transitions to the Fermi level. These responses may be strong even if the final and initial bands are not parallel. Further, these transitions may or may not be localized in $\overrightarrow{\mathrm{k}}$ space. As will be demonstrated, the strongest thermoresponse in gold is due to transitions in a large area of the Brillouin zone.

\section{INTERPRETATION OF OPTICAL EXPERIMENTS ON GOLD}

Following the rules of interpretation given in Sec. IV, we will now demonstrate how the optical experiments are related to the calculated band structure. In Sec. VA, the photoemission experiment is considered, and it seems reasonable from this to conclude that, in gold, nondirect transitions are infrequent even from the $d$ bands. It is shown in Sec. $\mathrm{V} B$ that an attempt at interpreting the results of static reflectance in terms of critical-point transitions fails completely although the calculated $\epsilon_{2}^{(i)}(\omega)$

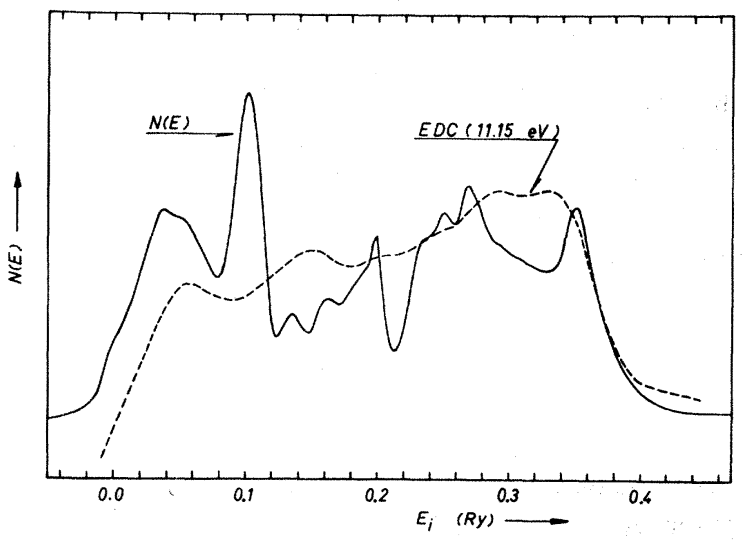

FIG. 15. Calculated (broadened) density of states and ED curve from photoemission experiment. 
agrees well with the experimental quantity that correlates to the joint density of states. Structure elements in the modulated reflectance spectra are interpreted in Sec. V C in terms of the present band model.

\section{A. Photoemission}

In Fig. 15, the experimental ${ }^{43}$ electron distribution (ED) curve corresponding to the photon energy $\hbar \omega=11.15 \mathrm{eV}$ is plotted together with the one-electron density-of-states function derived from the RAPW band structure. The latter function is identical with the one shown in Fig. 8 apart from the fact that the energy levels have been broadened by a Lorentzian with half-width $\Gamma=0.1 \mathrm{eV}$. The width of the $d$ band cannot be derived from the experimental ED curve in Fig. 15 because the photon energy was not large enough to allow excitation of states at the bottom of the $d$ band. Higher photon energies were used in the measurements by Eastman and Cashion, ${ }^{44}$ and they obtained for the $d$ bandwidth the value $5.7 \pm 0.3 \mathrm{eV}$. This agrees well with our value $(5.8 \mathrm{eV})$.

The positions of the peaks in the two curves are almost uncorrelated, and further, it was found in the experiment that some of the peaks moved when the photon energy was changed. ${ }^{43,45}$ Therefore we must conclude that although the gross features of the ED curves are reminiscent of those of the oneelectron density-of-states function, comparison of the detailed structure in the two curves demonstrates that the nondirect model does not apply for most of the transitions. We have therefore calculated ${ }^{41}$ the energy distribution of joint density of states [Eq. (12)], i. e. , the function that in the direct model predicts the peak positions of the ED curve for a given photon energy $\hbar \omega$. This function for $\hbar \omega=10.6$ $\mathrm{eV}$ is shown in Fig. 16 together with the ED curve

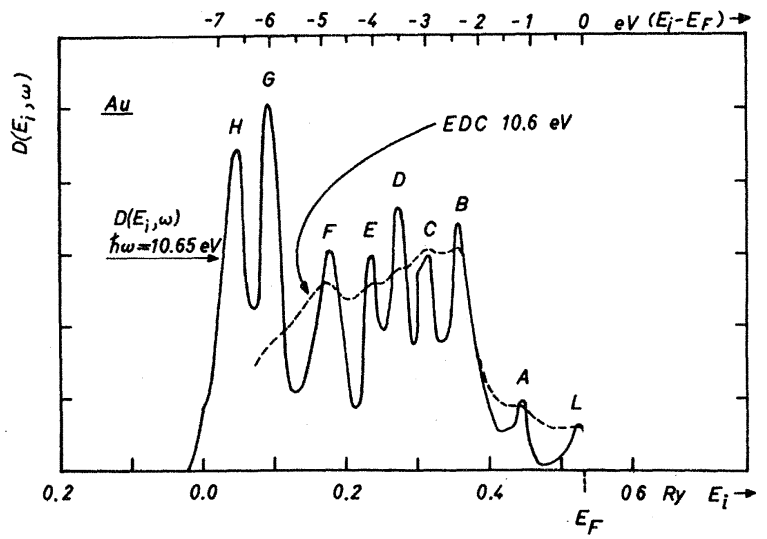

FIG. 16. Photoemission ED curve [experiment by Nilsson et al. (Ref. 43)] plotted vs initial-state energy together with $D\left(E_{i}, \omega\right)$, the energy distribution of joint density of states (direct model).

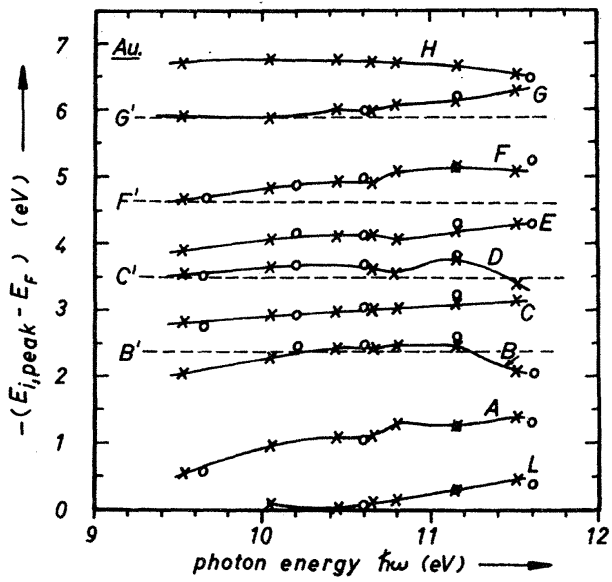

FIG. 17. Variation of spectral position (initial-state energy) of the peaks in $D\left(E_{i}, \omega\right)$ and the ED curves vs photon energy $\hbar \omega$ (direct model). Broken lines are peak positions predicted by a nondirect model:

corresponding to almost the same photon energy. There are eight peaks in the ED curve, and the same number of peaks appear in the calculated function $D\left(E_{i}, \omega\right)$ for $E_{i}$ in the range of initial state energies covered by the photoemission experiment. All peaks agree in spectral position.

This agreement is not unique to the photon energy $10.6 \mathrm{eV}$. Figure 17 shows that, when $\hbar \omega$ is varied, all theoretical and experimental peak positions follow each other. The same figure shows how a nondirect model would predict the positions of peaks $B^{\prime}, C^{\prime}, F^{\prime}$, and $G^{\prime}$. The nondirect model predicts the correct peak positions only in very small ranges of photon energy.

We thus conclude that it is not necessary to invoke the concept of nondirect transitions in an analysis of photoemission data for gold. The photoemission spectrum can be described in terms of a

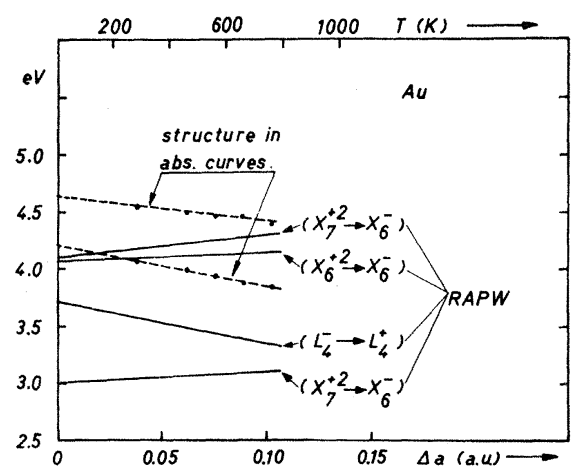

FIG. 18. Broken lines: elements of structure in the static absorption curves (Ref. 10). Full lines: RAPW band separations for varying lattice parameter. 


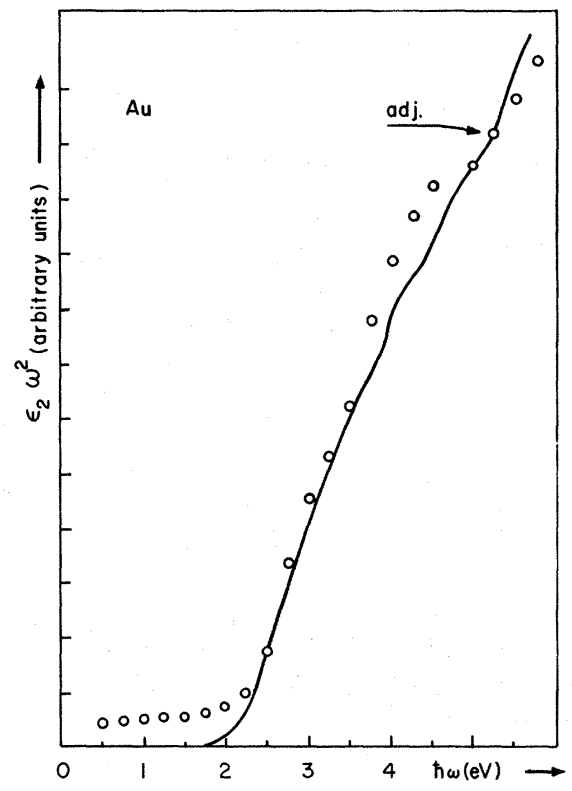

FIG. 19. $\epsilon_{2}(\omega) \cdot \omega^{2}$ of gold as obtained by Pells and Shiga (Ref. 10) from their polarimetric measurements at $T=295 \mathrm{~K}$ (circles). The joint density-of-states function obtained from the RAPW calculation is plotted as a full line and is made to coincide with the experimental point at $5 \mathrm{eV}$, with no adjustment for slope.

model assuming nondirect transitions to have negligible occurrence when compared to the number of direct transitions. This is somewhat in disagreement with the conclusions of Krolikowski and Spicer. ${ }^{46}$ However, their arguments are based on ED curves with fewer elements of structure (lower resolution) than contained in the curves obtained by Nilsson et al. ${ }^{43}$ To compare the direct model to the ED curves of Ref. 46 we would have to introduce a considerable level broadening. In fact this would to some extent make the gross features of $D\left(E_{i}, \hbar \omega\right)$ and $N\left(E_{i}\right)$ similar. But there are still peaks in $D\left(E_{i}, \hbar \omega\right)$ that are not in $N\left(E_{i}\right)$. The reason we can rule out the nondirect model is that we are able to make a detailed comparison between theory and experiment. Results similar to ours have been obtained for $\mathrm{Cu}^{47}$ and $\mathrm{Pd} .{ }^{48}$

\section{B. Static Reflectance}

The static reflectance spectrum of gold has been recorded by several authors. ${ }^{6,49,50}$ In a recent polarimetric work over an extended temperature range, the present author $\mathrm{s}^{10}$ interpret their results in terms of critical-point transitions. Guided by the qualitative features derived from nonrelativistic calculations, Pells and Shiga ${ }^{10}$ assign the two most prominent structure elements $(4.6$ and $4.1 \mathrm{eV})$ to transitions $X_{5} \rightarrow X_{4}^{\prime}$ and $L_{2}^{\prime} \rightarrow L_{1}^{u}$. In the experiment, both elements of structure show negative temperature coefficients.

In Fig. 18, we have plotted the spectral position of the observed structure elements as a function of temperature together with the calculated energy separations relevant for a check of the above-mentioned interpretation. The temperature shifts were calculated as described in Secs. IIID and III E. It is seen that the energies do not agree, and further, all the calculated $X$ transitions in Fig. 18 exhibit temperature coefficients opposite to the ones observed for the elements of structure in the absorption. By searching the whole Brillouin zone for

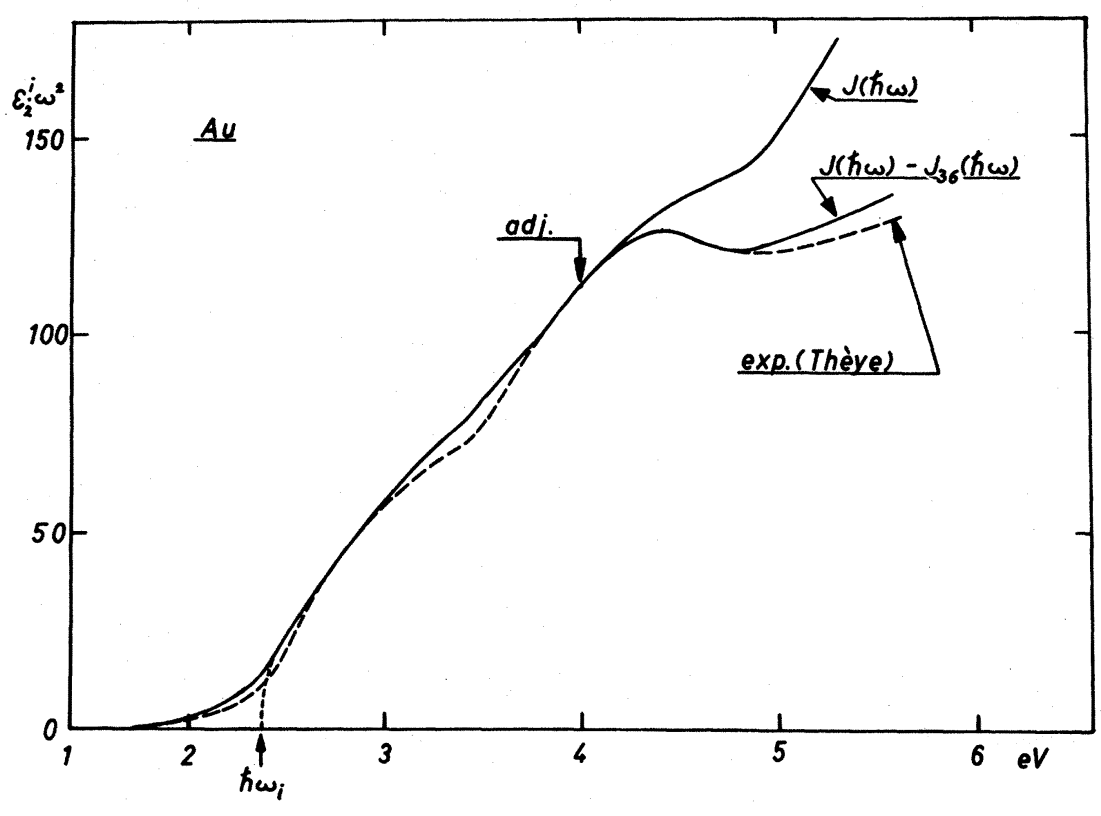

FIG. 20. $\epsilon_{2}^{(i)}(\omega) \cdot \omega^{2}$ as obtained by Thèye (Ref. 50 ) together with the calculated $J(\hbar \omega)$ and $J(\hbar \omega)-J_{36}(\hbar \omega)$. 


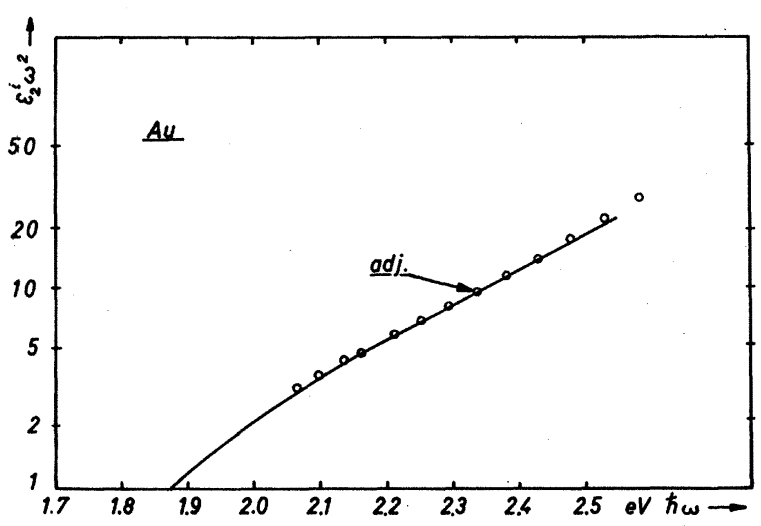

FIG. 21. Logarithmic plot of $\epsilon_{2}^{(i)}(\omega) \cdot \omega^{2}$ in the tail region. Circles indicate the experimental results (Ref. $50)$.

critical points, we found several but none with a gap in the energy range of interest here. Thus, it was not possible to find in the calculated band structure any critical points matching the observed structure in the static curves. This result is completely in line with the discussion in Sec. IV. Sommers and $\mathrm{Amar}^{23}$ report similar discrepancies, but they call in many-body effects and phonon-assisted transitions as a possible explanation.

In a crude model we can calculate $\epsilon_{2}^{(i)}(\omega)$, which is also the result of the experiment when the Drude term has been subtracted. We assume all matrix elements to be constant and use therefore the relation (15). Figure 19 shows the calculated joint density-of-states function and $\epsilon_{2}(\omega) \omega^{2}$ as derived from the experiments by Pells and Shiga $(T=295 \mathrm{~K})$ in the energy range $0-6 \mathrm{eV}$. The two curves are adjusted at $5 \mathrm{eV}$. We have chosen a point of adjustment for unknown parameters far from the interband edge since the Drude ter $\mathrm{m}^{49}$ has not been subtracted from the experimental curve. It is seen that with only one point of adjustment the theoretical curve even in this crude model follows the experimental one fairly well. In Figs. 20 and 21, the calculated $\epsilon_{2}^{(i)}(\omega) \omega^{2}$ function is compared to the experiment by Thèye. ${ }^{50}$ The deviations between the experimental and theoretical results around $5 \mathrm{eV}$ probably are due to our assumption about the constant matrix elements. If we take out the partial

TABLE VI. Comparison of the calculated interband edge $\left(\hbar \omega_{i}\right)$ with experimental results.

\begin{tabular}{lcccc}
\hline \hline & $\begin{array}{c}\text { Cooper } \\
\text { et al. } \\
\text { (Ref. 6) }\end{array}$ & $\begin{array}{c}\text { Pells } \\
\text { and Shiga } \\
\text { (Ref. 10) }\end{array}$ & $\begin{array}{c}\text { Thèye } \\
\text { (Ref. 50) }\end{array}$ & $\begin{array}{c}\text { Present } \\
\text { calc. }\end{array}$ \\
\hline $\begin{array}{l}\hbar \omega_{i} \\
(\mathrm{eV})\end{array}$ & 2.35 & 2.4 & 2.45 & 2.38 \\
\hline \hline
\end{tabular}

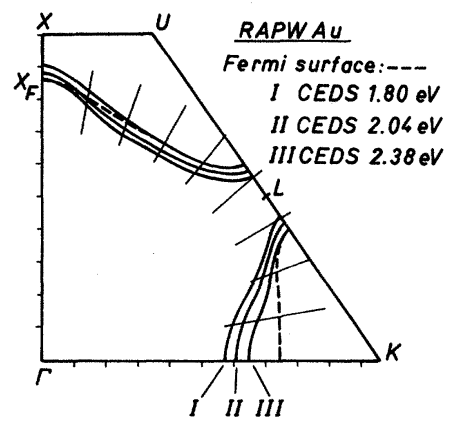

FIG. 22. (110) sections of CEDS and Fermi surface.

joint density-of-states function corresponding to transitions from band 3 to band 6 , the calculated curve follows nicely the experimental curve. However, relative weights cannot be attributed to the different transitions before the calculation of the matrix elements has been completed. ${ }^{51}$ Further, it cannot be excluded that excitation of surface plasmons ${ }^{52}$ may cause structure in the experimental curve around $5 \mathrm{eV}$.

The behavior of $\epsilon_{2}^{(i)}(\omega)$ in the energy range near the interband edge $\hbar \omega=\hbar \omega_{i}$ is particularly interesting. The edge is often assumed to be well defined. However, below $\omega_{i}$ the $\epsilon_{2}^{(i)} \omega^{2}$ function has a tail that is well described as being exponentially dependent on $\omega$. This tail is also present in our calculation, and thus it is not due to many-body effects, level broadening etc., but simply reflects the band structure. In order to follow conventions from experimental work we define the edge energy $\hbar \omega_{i}$ as being the spectral position of the parabolic footpoint (see Fig. 20) of the $\epsilon_{2}^{(i)}(\omega) \omega^{2}$ function. In Table VI, we compare our theoretical value of $\hbar \omega_{i}$ to experimental results. The tail $\epsilon_{2}^{(i)}(\omega) \omega^{2}$ below the edge is shown in the logarithmic plot in Fig. 21.

The shape of the function $\epsilon_{2}^{(i)}(\omega)$ at and below the interband edge is entirely determined by bands 5 and 6 . This can be seen from the plots of the partial joint density-of-states functions (Fig. 9). For photon energies less than $\hbar \omega=1.7 \mathrm{eV}$ no final states above the Fermi level can be reached, and $\epsilon_{2}^{(i)}(\omega)$ is zero. When the photon energy is increased, the surfaces of constant energy difference, $E_{f}-E_{i}=\hbar \omega$, first touch the Fermi surface at the points $X_{F}$ ( $\Gamma-X$ direction) (see Fig. 22), and then for larger $\hbar \omega$ they cut through the Fermi surface. However, as long as $\hbar \omega<\hbar \omega_{i}$, only few final states are available since only a small portion of the constant energy difference surface (CEDS) is outside the Fermi surface. Thus the tail absorption is caused by transitions between states corresponding to $\vec{k}$ being near the $X_{F}$ points.

Figures 22 and 23 show that when the photon energy is increased to $2.38 \mathrm{eV}$ the corresponding 


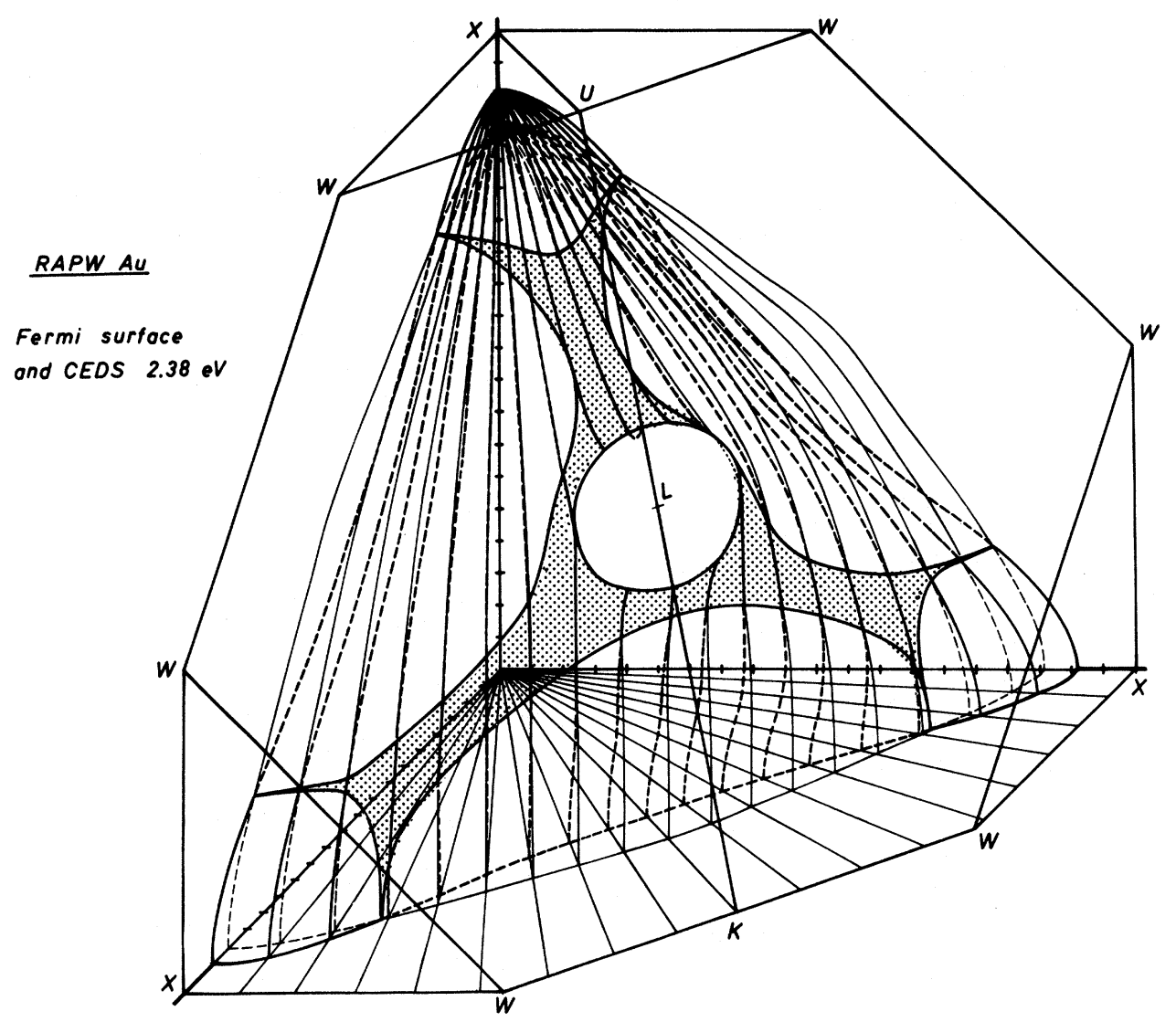

FIG. 23. Axiometric plot of sections of the Fermi surface (fine lines) and the CEDS corresponding to $E_{F}-E_{i}=\hbar \omega_{i}$ $=2.38 \mathrm{eV}$ (heavy lines) for constant azimuthal angles. In the hatched area, the two surfaces coincide. $\overrightarrow{\mathrm{k}}$ vectors in this region correspond to the states giving the steep increase of the absorption at the interband edge $\hbar \omega_{i}=2.38 \mathrm{eV}$.

CEDS coincides with the Fermi surface over a large area, and thus suddenly very many unoccupied final states become accessible for $\hbar \omega \geq 2.38 \mathrm{eV}$. We are then able to determine the states in $\vec{k}$ space that give rise to the steep increase in the absorption at $\hbar \omega=\hbar \omega_{i}=2.38 \mathrm{eV}$. These states are shown by the hatching in Fig. 23.

\section{Modulated Reflectance}

Several modulated reflectance spectra of gold are available. The largest spectral range is covered by the thermoreflectance measurement (at $120 \mathrm{~K}$ ) by Scouler, ${ }^{53}$ followed by Feinleib's ${ }^{54}$ electroreflectance spectrum and the piezoreflectance measurement by Garfinkel et al. , ${ }^{55}$ the latter two recorded at $300 \mathrm{~K}$.

Figure 24 shows the piezoreflectance and thermoreflectance responses $\Delta R / R$ as a function of photon energy, and Fig. 25 shows the $\Delta \epsilon_{2}$ spectrum on which we based our interpretation. $\Delta \epsilon_{2}(\omega)$ was derived from the thermoreflectance spectrum $^{53}$ by Kramers-Kronig analysis. ${ }^{56}$ The arrows indicate the spectral positions of critical-point and Fermi- level transitions as derived from the RAPW calculation.

From the discussion (Sec. V B) of the interband edge and the sudden increase in number of final states (Fig. 23) it is obvious that a large thermoresponse at $\hbar \omega=\hbar \omega_{i}=2.38 \mathrm{eV}$ should be expected. The temperature modulation will cause the Fermi surface and the CEDS $\left(E_{f}-E_{i}=\hbar \omega\right)$ to oscillate slightly relative to each other. In fact, the experimental spectrum contains a large response at 2.4 eV. This element of structure in the spectrum is then due to transitions at points $\vec{k}$ on the hatched area of the Fermi surface (Fig. 23). The full assignment of the structure elements in the $\Delta \epsilon_{2}$ spectrum is listed in Table VII and further illustrated by Fig. 25. Seven structure elements correspond to Fermi-level transitions. The remaining six structure elements in Table VII are assigned to critical-point transitions. It should be emphasized that a search in the entire Brillouin zone for critical points left the assignment in Table VII as the only possible. The consistency of the present interpretation is further demonstrated by considering 
TABLE VII. Structure in $\Delta \epsilon_{2}$ determined from thermoreflectance and its assignment to band separations calculated by the RAPW method (Table II, Figs. 5 and 22-24).

\begin{tabular}{|c|c|c|c|}
\hline \multicolumn{2}{|c|}{ Energies (eV) } & \multirow{2}{*}{\multicolumn{2}{|c|}{ Assignment }} \\
\hline Expt. & RAPW & & \\
\hline 2.4 & 2.38 & $(1)$ & band $5 \rightarrow$ band $6\left(E_{F}\right)$ (see Fig. 23 ) \\
\hline 2.9 & 2.92 & $(2)$ & $\begin{array}{l}\text { Fermi-level transitions, e.g. } \\
\Delta_{6}^{2} \text { or } \Delta_{7}^{2} \rightarrow E_{F}\left(\Delta_{6}^{3}\right)\end{array}$ \\
\hline 3.2 & 3.21 & (3) & $\begin{array}{l}\text { band } 4 \rightarrow \text { band } 6 E_{F} \text {, e.g. } \\
\left(Q_{3}+Q_{4}\right)^{4} \rightarrow E_{F}\left(Q_{3}+Q_{4}\right)\end{array}$ \\
\hline 3.6 & 3.71 & (4) & $L_{4}^{-} \rightarrow L_{4}^{+}$ \\
\hline 4.1 & 4.10 & (5) & $X_{7}^{+2} \rightarrow X_{6}^{-}$ \\
\hline $\begin{array}{l}4.6 \\
\cdots\end{array}$ & $\ddot{4}$ & (6) & $\begin{array}{l}\text { third } d \text { band } \rightarrow E_{F} \\
\text { e.g., }\left(Q_{3}+Q_{4}\right)^{3} \rightarrow E_{F}\end{array}$ \\
\hline 5.6 & 5.7 & & $\begin{array}{l}\text { second } d \text { band } \rightarrow E_{F} \text {, e.g., } \\
\text { near }(3,5,2) \pi / 4 a\end{array}$ \\
\hline $\begin{array}{l}6.1 \\
\cdots\end{array}$ & $\ddot{6.18}$ & (7) & $\begin{array}{l}\text { lowest } d \text { band } \rightarrow E_{F} \text {, e.g., } \\
\Sigma_{5}^{1} \rightarrow E_{F}\left(\Sigma_{5}^{6}\right)\end{array}$ \\
\hline 7.2 & 7.15 & $(8)$ & $W_{7}^{3} \rightarrow W_{6}^{3}$ \\
\hline 8.0 & 8.08 & (9) & $W_{7}^{3} \rightarrow W_{7}^{4}$ \\
\hline 8.9 & 8.98 & (10) & $X_{6}^{+1} \rightarrow X_{6}^{-}$ \\
\hline 9.3 & 9.37 & (11) & $W_{7}^{3} \rightarrow W_{6}^{4}$ \\
\hline 9.7 & 9.68 & (12) & $\Sigma_{5}^{5} \rightarrow \Sigma_{5}^{8}$ \\
\hline
\end{tabular}

the structure elements at 3.6 and $4.1 \mathrm{eV}$ in the experimental trace. These we have assigned to transitions at the points $L$ and $X$, namely, $L_{4}^{-}-L_{4}^{+}$and $X_{7}^{+2} \rightarrow X_{6}^{-}$(Figs. 5 and 26). When calculating the strain responses, we found these energy gaps to be particularly sensitive to strain (Fig. 18). It is therefore a strong support for our interpretation that particularly large piezoresponses are observed at these energies (Fig. 24).

The transition $X_{3} \rightarrow X_{4}^{\prime}$ is forbidden. When spinorbit coupling is introduced, the number of differ-

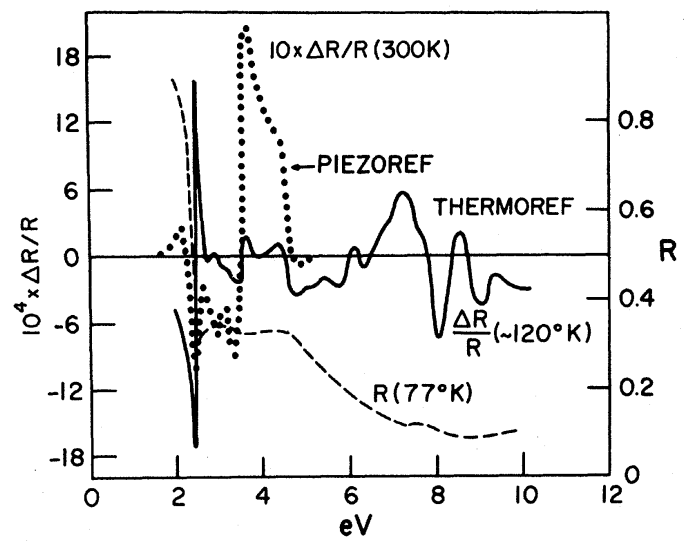

FIG. 24. $\Delta R / R$ obtained from piezo- and thermoreflectance of gold (Refs. 53 and 55) (after Cardona, Ref. 56).

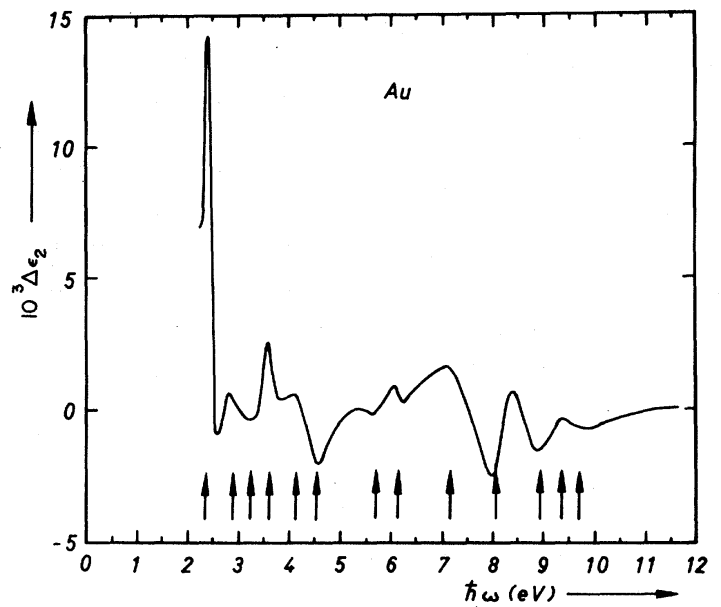

FIG. 25. $\Delta \epsilon_{2}$ obtained by Kramers-Kronig analysis (Ref. 56) of the thermoreflectance spectrum (Ref. 53). Arrows indicate the spectral positions of Fermi-level and critical-point transitions as derived from the RAPW band calculation. Assignments are given in Table VII.

ent irreducible representations is decreased, and the transition corresponding to $X_{3} \rightarrow X_{4}^{\prime}$ becomes allowed. However, since it is forbidden in the nonrelativistic case, it is reasonable to assume the matrix element to be small. We therefore attempted to perform the interpretation of the modulated reflectance experiments without including this transition.

Note that in the experimental column of Table VII the margin of $0.1 \mathrm{eV}$ for the spectral location of structure in $\Delta \epsilon_{2}$ is rather wide. Defining the energy of structure to better than $0.1 \mathrm{eV}$ would ignore the notorious difficulties that the line-shape interpretation of modulated spectra encounters. With our present knowledge of the basic modulation mechanism, we cannot decide whether the critical-

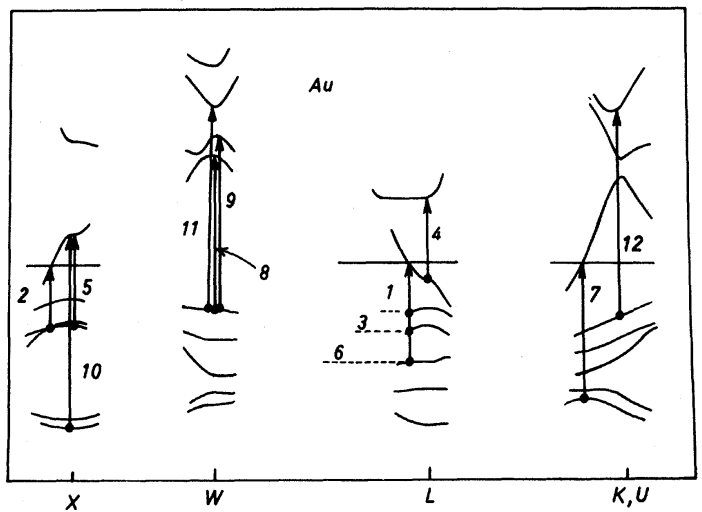

FIG. 26. Critical-point transitions and representatives of Fermi-level transitions entering the present interpretation of the modulated reflectance spectra of gold. 
point arrow is correlated to a peak, a dip, or an inflection point. The value of the agreement must be seen in the gapless coincidence of the first 13 critical-point and Fermi-level energies on the one hand, and on a sequence of structure elements on the other hand, with respect to their relative spectral location and their energy differences, given the cautious margin of $0.1 \mathrm{eV}$ for the definition of structure.

\section{CONCLUSION}

Although ambiguity exists in the construction of the muffin-tin potentials, it is with some satisfaction that we conclude that the method of constructing a silver potential which on many accounts has proved to give a band structure in close agreement with experiments applies equally successfully to gold.

Whereas copper, and to some extent silver, can be considered in the nonrelativistic scheme, the relativistic effects in the band structure of gold are large. By comparing APW and RAPW band calculations of gold, we found shifts of the same order of magnitude as the band gaps. Therefore, it was important to apply the relativistic scheme in this work where a quantitative interpretation of optical experiments was performed.

Establishing the rules of interpretation of the optical data, it was important to discuss the possible effects of localization of the $d$ states. It was found that in gold the $d$ states can be well described as being good band states; i.e., localization effects can be neglected. This implies that we could calculate the $d$ band in the same way as the broad conduction band. Further, the fact that the $d$ band also is broad enabled us to relate the calculated energy gaps directly to the observed excitation energies in the same way as given by Koopmans's theorem.

The consistency of the whole interpretation of the optical experiments is in itself a support for the conclusion about the localization effects being small in gold. This interpretation could be made in terms of direct transitions only although we realize that there may be, even in the photoemission experiment, difficulties in distinguishing between direct and nondirect transitions. The calculated values of the critical parameters, width and position of the $d$ band, agree with the results obtained from photoemission. But our comparison between the experimental ED curves and the theoretical model is so detailed that we can conclude that it is not at the present stage necessary to involve the concept of nondirect transitions. The calculated spectral position of the interband edge deviates only a few hundredths of an $\mathrm{eV}$ from the observations in static as well as modulated reflectance experiments. Also the tail of $\epsilon_{2}^{(i)}(\omega)$ below the edge is accounted for in our model. By calculating constant energy difference surfaces we saw that the absorption corresponding to the tail occurs between states having $\overrightarrow{\mathrm{k}}$ close to the $\Gamma-X$ points at the Fermi surface. The edge transitions occur at an area where the CEDS $\left(\Delta E=\hbar \omega=\hbar \omega_{i}=2.38 \mathrm{eV}\right)$ coincides with the Fermi surface. This region has been traced out.

The interpretation of static and modulated reflectance in terms of our band model establishes firm guidelines for the analysis of such spectra: $\epsilon_{2} \omega^{2}$ clearly relates to a calculated density-of-states profile. Attempts to fit structure in $\epsilon_{2}$ to critical points fail with respect ot energy value and temperature coefficient. A sequence of structure elements in $\Delta \epsilon_{2}$, however, correlates remarkably well with a series of critical-point and Fermi-level gaps. Such proper interpretation eliminates problems encountered in all previous band calculations of gold.

\section{ACKNOWLEDGMENTS}

The authors greatly acknowledge informative discussions with P.-O. Nilsson, L. Walldèn, and C. Norris on the photoemission experiments. Professor A. R. Mackintosh and O. K. Andersen supplied the atomic charge densities calculated by Liberman et al. and some of their RAPW computer codes. G. B. Jensen provided the results of his careful study of numerical methods of calculating spherical Bessel functions. Thanks are due Professor K. Særmark for his continuous interest in the work. One of us (B.O.S.) acknowledges gratefully the hospitatility of the Technical University of Denmark, in particular the Physics Laboratory I during a sabbatical year ending in fall 1970. $\dagger$ Partially based on a thesis submitted to the Technical University of Denmark in partial fulfillment of the requirements for the Lic. Techn. Degree.

${ }^{1}$ J. M. Ziman, Proc. Phys. Soc. (London) $\underline{91}, 701$ (1967).

${ }^{2}$ R. E. Watson, H. E. Ehrenreich, and L. Hodges, Phys. Rev. Letters 24, 829 (1970).

${ }^{3}$ C. N. Berglund and W. E. Spicer, Phys. Rev. 136, A1044 (1964).

${ }^{4}$ W. E. Spicer, Phys. Rev : 154, 385 (1967).
${ }^{5}$ S. Doniach, Phys. Rev. B $\underline{2}, 3898$ (1970).

${ }^{6}$ B. R. Cooper, H. Ehrenreich, and H. R. Philipp, Phys. Rev. 138, A494 (1965).

${ }^{7}$ E. O. Kane, Phys. Rev. 146, 558 (1966).

${ }^{8}$ F. Herman, R. L. Kortum, C. D. Kuglin, and R. A. Short, Quantum Theory of Atoms, Molecules, Solid State (Academic, New York, 1966), p. 381.

${ }^{9}$ N. E. Christensen and B. O. Seraphin, Solid State Commun. 8 , 1221 (1970).

${ }^{10}$ G. P. Pells and M. Shiga, J. Phys. C 2 , 1835 (1969). 
${ }^{11}$ D. Liberman, J. T. Waber, and D. T. Cromer, Phys. Rev. 137, A27 (1965).

${ }^{12}$ N. E. Christensen, Phys. Status Solidi 31, 635 (1969).

${ }^{13}$ J. O. Henningsen, Phys. Status Solidi 32,239 (1969); 7, 763 (1969); Phys. Rev. Letters 24, 823 (1970); J. O. Henningsen and D. S. Falk, Phys. Rev. Letters 26, 1174 (1971); D. S. Falk, J. O. Henningsen, H. L. Skriver, and N. E. Christensen, Phys. Rev. B (to be published).

${ }^{14}$ R. A. Ballinger and C. A. W. Marshall, J. Phys. C 2,1822 (1969).

${ }^{15}$ S. Kupratakuln and G. C. Fletcher, J. Phys, C $\underline{2}$, 1886 (1969).

${ }^{16}$ F. Herman and S. Skillman, Atomic Structure Calculations (Prentice-Hall, Englewood Cliffs, N.J., 1963).

${ }^{17}$ W. J. O'Sullivan, A. C. Switendick, and J. E. Schirber, Phys. Rev. B 1,1443 (1970).

${ }^{18}$ R. L. Jacobs, J. Phys. C 1 , 1296 (1968).

${ }^{19} \mathrm{~T}$. Loucks, Augumented Plane Wave Method (Benjamin, New York, 1967).

${ }^{20}$ O. K. Andersen, Phys. Rev. B 2,883 (1970).

${ }^{21}$ D. Koelling, MIT Solid State Mol. Theory Group Quart. Progr. Rept. 68, 36 (1968).

${ }^{22}$ M. E. Rose, Relativistic Electron Theory (Wiley,

New York, 1961).

${ }^{23}$ C. B. Sommers and H. Amar, Phys. Rev. 188,1117 (1969).

${ }^{24}$ S. Kupratakuln, J. Phys. F $\underline{3}, 109$ (1970).

${ }^{25} \mathrm{~J}$. W. D. Conolly and K. H. Johnson, MIT Solid State Mol. Theory Group Semi-Annual Progr. Rept. $\underline{72}, 19$ (1970).

${ }^{26}$ M. G. Ramchandani, J. Phys. F $\underline{3}, 1$ (1970).

${ }^{27} \mathrm{H}$. Ehrenreich and L. Hodges, in Methods in Computational Physics, edited by B. Alder, S. Fernbach, and M. Rotenberg (Academic, New York, 1968), Vol. 8, p. 149.

${ }^{28}$ G. Gilat and L. J. Raubenheimer, Phys. Rev. 144, 390 (1966).

${ }^{29}$ N. E. Christensen (unpublished).

${ }^{30}$ D. L. Martin, Phys. Rev. $\underline{170}, 650$ (1968).

${ }^{31}$ N. E. Christensen, Solid State Commun. 9 , 749 (1971).

${ }^{32}$ J. P. Walter, R. R. L. Zucca, M. L. Cohen, and Y. R. Shen, Phys. Rev. Letters 24, 102 (1970).

${ }^{33}$ In fact this is the square root of the Debye-Waller factor known from $x$-ray diffraction, where the thermal vibrations reduce to intensities by $e^{-2 W}$.

${ }^{34} \mathrm{~J}$. M. Ziman, Principles of the Theory of Solids

(Cambridge U.P., Cambridge, England, 1964).

${ }^{35}$ P. K. Sharma and S. K. Joshi, J. Chem. Phys. $\underline{39}$, 2633 (1963); 40, 662 (1964).

${ }^{36}$ R. P. Gupta, J. Phys. Soc. Japan 21, 1726 (1966).

${ }^{37} \mathrm{~J}$. R. Neighbours and G. A. Alers, Phys. Rev. 111, 707 (1958).

${ }^{38}$ J. C. Slater, MIT Solid State Mol. Theory Group Semi-Annual Progr. Rept. 71, 3 (1969).

${ }^{39}$ R. Y. Koyama and N. V. Smith, Phys. Rev. B $\underline{2}$, 3049 (1970).

${ }^{40}$ N. E. Christensen, Ph. D. thesis (The Technical University of Denmark, 1970) (unpublished).

${ }^{4 i}$ N. E. Christensen, Phys. Letters 35A, 206 (1971).

${ }^{42}$ B. O. Seraphin, in Semiconductors and Semimetals, edited by R. K. Willardson and A. Beer (Academic, New York, to be published), Vol. VI.

${ }^{43}$ P. -O. Nilsson, C. Norris, and L. Wallden, Solid State Commun. 7, 1705 (1969).

${ }^{44}$ D. E. Eastman and J. K. Cashion, Phys. Rev. Letters 24,310 (1970).

${ }^{45} \mathrm{P} .-\mathrm{O}$. Nilsson, C. Norris, and L. Wallden, Physik

Kondensierten Materie 11, 220 (1970).

${ }^{46}$ W. F. Krolikowski and W. E. Spicer, Phys. Rev. 185, 882 (1969).

${ }^{47}$ N. V. Smith, Phys. Rev. Letters 23,1452 (1969).

${ }^{48} \mathrm{~J}$. F. Janak, D. E. Eastman, and A. R. Williams, Solid State Commun. $\underline{8}, 271$ (1970).

${ }^{49} \mathrm{H}$. E. Bennett and J. M. Bennett, in Optical Properties and Electronic Structure of Metals and Alloys, edited by F. Abeles (North-Holland, Amsterdam, 1966), p. 285.

${ }^{50}$ M. -L. Théye, Phys. Rev. B 2 , 3060 (1970).

${ }^{51}$ Page 175 of Ref. 49.

${ }^{52} \mathrm{H}$. E. Bennett called our attention to this possibility (private communication).

${ }^{53}$ W. J. Scouler, Phys. Rev. Letters 18,445 (1967).

${ }^{54} \mathrm{~J}$. Feinleib, Phys. Rev. Letters 16, 1200 (1966).

${ }^{55} \mathrm{M}$. Garfinkel, J. J. Tiemann, and W. E. Engeler, Phys. Rev. 148, 695 (1966).

${ }^{56} \mathrm{M}$. Cardona, in Solid State Physics, edited by F. Seitz, D. Turnbull, and H. Ehrenreich (Academic, New York, 1969), Suppl. 11, p. 130. 